\title{
Quadratic life span of periodic gravity-capillary water waves
}

\author{
M. Berti, R. Feola, L. Franzoi
}

\begin{abstract}
We consider the gravity-capillary water waves equations for a bi-dimensional fluid with a periodic one-dimensional free surface. We prove a rigorous reduction of this system to Birkhoff normal form up to cubic degree. Due to the possible presence of 3 -waves resonances for general values of gravity, surface tension and depth, such normal form may be not trivial and exhibit a chaotic dynamics (Wilton-ripples). Nevertheless we prove that for all the values of gravity, surface tension and depth, initial data that are of size $\varepsilon$ in a sufficiently smooth Sobolev space lead to a solution that remains in an $\varepsilon$-ball of the same Sobolev space up times of order $\varepsilon^{-2}$. We exploit that the 3 -waves resonances are finitely many, and the Hamiltonian nature of the Birkhoff normal form.
\end{abstract}

\section{Introduction and main results}

We consider an incompressible and irrotational perfect fluid, under the action of gravity, occupying at time $t$ the bi-dimensional domain

$$
\mathcal{D}_{\eta}:=\{(x, y) \in \mathbb{T} \times \mathbb{R} ;-h<y<\eta(t, x)\}, \quad \mathbb{T}:=\mathbb{R} /(2 \pi \mathbb{Z}),
$$

periodic in the horizontal variable, with depth $h$ which may be finite or infinite. The timeevolution of the fluid is determined by a system of equations for the free surface $\eta(t, x)$ and the function $\psi(t, x):=\Phi(t, x, \eta(t, x))$ where $\Phi$ is the velocity potential in the fluid domain. Given the shape $\eta(t, x)$ of the domain $\mathcal{D}_{\eta}$ and the Dirichlet value $\psi(t, x)$ of the velocity potential at the top boundary, one recovers $\Phi(t, x, y)$ as the unique solution of the elliptic problem

$$
\Delta \Phi=0 \text { in } \mathcal{D}_{\eta}, \quad \partial_{y} \Phi=0 \text { at } y=-h, \quad \Phi=\psi \text { on }\{y=\eta(t, x)\} .
$$

According to Zakharov [35] and Craig-Sulem [13], the $(\eta, \psi)$ variables evolve under the system

$$
\left\{\begin{array}{l}
\partial_{t} \eta=G(\eta) \psi \\
\partial_{t} \psi=-g \eta-\frac{1}{2} \psi_{x}^{2}+\frac{1}{2} \frac{\left(\eta_{x} \psi_{x}+G(\eta) \psi\right)^{2}}{1+\eta_{x}^{2}}+\kappa \partial_{x}\left(\frac{\eta_{x}}{\left(1+\eta_{x}^{2}\right)^{\frac{1}{2}}}\right)
\end{array}\right.
$$

where $g>0$ is the acceleration of gravity, $\kappa>0$ the surface tension, and $G(\eta)$ is the nonlocal Dirichlet-Neumann operator defined by $G(\eta) \psi:=\left(\partial_{y} \Phi-\eta_{x} \partial_{x} \Phi\right)(t, x, \eta(t, x))$. 
As observed by Zakharov [35], the equations (1.1) are the Hamiltonian system

$$
\partial_{t} \eta=\nabla_{\psi} H(\eta, \psi), \quad \partial_{t} \psi=-\nabla_{\eta} H(\eta, \psi),
$$

where $\nabla$ denotes the $L^{2}$-gradient, with Hamiltonian

$$
H(\eta, \psi):=\frac{1}{2} \int_{\mathbb{T}} \psi G(\eta) \psi d x+\frac{g}{2} \int_{\mathbb{T}} \eta^{2} d x+\kappa \int_{\mathbb{T}} \sqrt{1+\eta_{x}^{2}} d x
$$

given by the sum of the kinetic and potential energy of the fluid and the energy of the capillary forces. We remind that the Poisson bracket between two functions $H(\eta, \psi), F(\eta, \psi)$ is

$$
\{H, F\}=\int_{\mathbb{T}}\left(\nabla_{\eta} H \nabla_{\psi} F-\nabla_{\psi} H \nabla_{\eta} F\right) d x .
$$

The "mass" $\int_{\mathbb{T}} \eta d x$ is a prime integral of $(1.1)$ and, with no loss of generality, we can fix it to zero by shifting the $y$ coordinate. Moreover (1.1) is invariant under spatial translations and Noether's theorem implies that the momentum $\int_{\mathbb{T}} \eta_{x}(x) \psi(x) d x$ is a prime integral of (1.1).

Let $H^{s}(\mathbb{T}):=H^{s}, s \in \mathbb{R}$, denote the Sobolev spaces of $2 \pi$-periodic functions of $x$. The variable $\eta$ belongs to the subspace $H_{0}^{s}(\mathbb{T})$ of $H^{s}(\mathbb{T})$ of zero average functions (for some positive $s$ ). On the other hand, the variable $\psi$ belongs to the homogeneous Sobolev space $\dot{H}^{s}(\mathbb{T}):=H^{s}(\mathbb{T}) / \sim$ obtained by the equivalence relation $\psi_{1}(x) \sim \psi_{2}(x)$ if and only if $\psi_{1}(x)-\psi_{2}(x)=c$ is a constant. This is coherent with the fact that only the velocity field $\nabla_{x, y} \Phi$ has physical meaning, and the velocity potential $\Phi$ is defined up to a constant. For simplicity we denote the equivalence class $[\psi]$ in $\dot{H}^{s}$ by $\psi$ and, since the quotient map induces an isometry of $\dot{H}^{s}(\mathbb{T})$ onto $H_{0}^{s}(\mathbb{T})$, we conveniently identify $\psi$ with a function with zero average.

The water waves equations 1.1 are a quasi-linear system. In the last years, they have been object of intense research both in the periodic setting $x \in \mathbb{T}^{d}, d=1,2$, and in the dispersive case $x \in \mathbb{R}^{d}$ with data decaying at infinity. A fundamental difference between these cases concerns the dynamical behavior of the linearized water waves equations at $(\eta, \psi)=0$. In $d=1$ they are

$$
\left\{\begin{array}{l}
\partial_{t} \eta=G(0) \psi \\
\partial_{t} \psi=-g \eta+\kappa \eta_{x x},
\end{array} \quad G(0)=\tanh (h D) D, \quad D:=\frac{1}{\mathrm{i}} \partial_{x}\right.
$$

with dispersion relation

$$
\Omega(\xi):=\Omega_{g, \kappa, h}(\xi):=\left(\kappa|\xi|^{3}+g|\xi|\right)^{\frac{1}{2}}(\tanh (h|\xi|))^{\frac{1}{2}} .
$$

Notice that, if $h=+\infty$, the Dirichlet-Neumann operator is $G(0)=|D|$ and the dispersion relation $\Omega(\xi)=\left(\kappa|\xi|^{3}+g|\xi|\right)^{\frac{1}{2}}$. In the case $x \in \mathbb{R}^{d}$, the solutions of (1.4) disperse to zero as $t \rightarrow+\infty$. On the contrary, if $x \in \mathbb{T}^{d}$, all the solutions of the linear system (1.4) are time periodic, or quasi-periodic, or almost periodic in time, with linear frequencies of oscillations $\Omega(j), j \in \mathbb{Z}^{d}$. In such a case a natural tool to analyze the nonlinear dynamics of (1.1), at least for small amplitude solutions, is normal form theory, that is particularly difficult due to the 
quasi-linear nature of the nonlinearity. In [14], Craig and Sulem developed a Birkhoff normal form analysis for (1.1) starting from the Taylor expansion of the Hamiltonian (1.2),

$$
H=H^{(2)}+H^{(3)}+H^{(\geq 4)},
$$

where (up to a constant)

$$
\begin{aligned}
H^{(2)} & :=\frac{1}{2} \int_{\mathbb{T}} \psi G(0) \psi d x+\frac{g}{2} \int_{\mathbb{T}} \eta^{2}+\frac{\kappa}{2} \int_{\mathbb{T}} \eta_{x}^{2} d x \\
H^{(3)} & :=\frac{1}{2} \int_{\mathbb{T}} \psi(D \eta D-G(0) \eta G(0)) \psi d x
\end{aligned}
$$

and $H^{(\geq 4)}$ collects all the terms of homogeneity in $(\eta, \psi)$ greater or equal than 4 . Unfortunately, in this Taylor expansion there is a priori no control of the unboundedness of the Hamiltonian vector field associated to $H^{(\geq 4)}$.

Normal form theory for gravity-capillary water waves, even in $x$, has been developed in Berti-Delort [6], proving, for most values of the parameters $(g, \kappa)$, an almost global existence result for the solutions of 1.1) in Sobolev spaces. A key point is, in analogy with the KAM theory approach in [8], to transform the unbounded water waves vector field to a paradifferential one with constant coefficient symbols, up to smoothing operators. Very recently, Birkhoff normal form and long time existence results for periodic pure gravity water waves in infinite depth, where no parameters are available, have been proved in Berti-Feola-Pusateri [7]. A key point is a normal form uniqueness argument which allows to identify the paradifferential normal form with the formal Hamiltonian Birkhoff normal form up to fourth degree, which turns out to be completely integrable.

Complementing these works, the goal of this paper is to prove that, for any value of $(\kappa, g, h), \kappa>0$, the gravity-capillary water waves system (1.1) is conjugated to its Birkhoff normal form, up to cubic remainders that satisfy energy estimates (Theorem 1.1), and that all the solutions of (1.1), with initial data of size $\varepsilon$ in a sufficiently smooth Sobolev space, exist and remain in an $\varepsilon$-ball of the same Sobolev space up times of order $\varepsilon^{-2}$, see Theorem 1.2. Let us state precisely these results.

Main results. To state our first main result, concerning the rigorous reduction of system (1.1) to its Birkhoff normal form up to cubic degree, let us assume that, for $s$ large enough and some $T>0$, we have a classical solution

$$
(\eta, \psi) \in C^{0}\left([-T, T] ; H_{0}^{s+\frac{1}{4}} \times \dot{H}^{s-\frac{1}{4}}\right)
$$

of the Cauchy problem for (1.1). The existence of such a solution, at least for small enough $T$, is guaranteed by local well-posedness theory, see the literature at the end of the section.

Theorem 1.1. (Cubic Birkhoff normal form) Let $\kappa>0, g \geq 0$ and $h \in(0,+\infty]$. There exist $s \gg 1$ and $0<\bar{\varepsilon} \ll 1$, such that, if $(\eta, \psi)$ is a solution of (1.1) satisfying (1.7) with

$$
\sup _{t \in[-T, T]}\left(\|\eta\|_{H_{0}^{s+\frac{1}{4}}}+\|\psi\|_{\dot{H}^{s-\frac{1}{4}}}\right) \leq \bar{\varepsilon},
$$


then there exists a bounded and invertible linear operator $\mathfrak{B}(\eta, \psi): H_{0}^{s+\frac{1}{4}} \times \dot{H}^{s-\frac{1}{4}} \rightarrow \dot{H}^{s}$, which depends (nonlinearly) on $(\eta, \psi)$, such that

$$
\begin{array}{r}
\|\mathfrak{B}(\eta, \psi)\|_{\mathcal{L}\left(H_{0}^{s+\frac{1}{4}} \times \dot{H}^{\left.s-\frac{1}{4}, \dot{H}^{s}\right)}\right.}+\left\|(\mathfrak{B}(\eta, \psi))^{-1}\right\|_{\mathcal{L}\left(\dot{H}^{s}, H_{0}^{s+\frac{1}{4}} \times \dot{H}^{s-\frac{1}{4}}\right)} \leq \\
1+C(s)\left(\|\eta\|_{H_{0}^{s+\frac{1}{4}}}+\|\psi\|_{\dot{H}^{s-\frac{1}{4}}}\right),
\end{array}
$$

and the variable $z:=\mathfrak{B}(\eta, \psi)[\eta, \psi]$ satisfies the equation

$$
\partial_{t} z=\mathrm{i} \Omega(D) z+\mathrm{i} \partial_{\bar{z}} H_{B N F}^{(3)}(z, \bar{z})+\mathcal{X}_{\geq 3}^{+}
$$

where:

(0) $\Omega(D)$ is the Fourier multiplier with symbol defined in (1.5) and $\partial_{\bar{z}}$ is defined in (5.3),

(1) the Hamiltonian $H_{B N F}^{(3)}(z, \bar{z})$ has the form

$$
H_{B N F}^{(3)}(z, \bar{z})=\sum_{\substack{\sigma_{1} j_{1}+\sigma_{2} j_{2}+\sigma_{2} j_{3}=0, \sigma_{i}= \pm, \sigma_{1} \Omega\left(j_{1}\right)+\sigma_{2} \Omega\left(j_{2}\right)+\sigma_{3} \Omega\left(j_{3}\right)=0, j_{i} \in \mathbb{Z} \backslash\{0\}}} H_{j_{1}, j_{2}, j_{3}}^{\sigma_{1}, \sigma_{2}, \sigma_{3}} z_{j_{1}}^{\sigma_{1}} z_{j_{2}}^{\sigma_{2}} z_{j_{3}}^{\sigma_{3}}
$$

where $z_{j}^{+}:=z_{j}, z_{j}^{-}:=\overline{z_{j}}$ and $z_{j}$ denotes the $j$-th Fourier coefficient of the function $z$ (see (2.2)), and the coefficients

$$
H_{j_{1}, j_{2}, j_{3}}^{\sigma_{1}, \sigma_{3}}:=\frac{\mathrm{i} \sigma_{2}}{8 \sqrt{\pi}}\left(\sigma_{1} \sigma_{3} j_{1} j_{3}+G_{j_{1}} G_{j_{3}}\right) \frac{\Lambda\left(j_{2}\right)}{\Lambda\left(j_{1}\right) \Lambda\left(j_{3}\right)}
$$

with $\Lambda(j)$ defined in (3.2) and $G_{j}:=\tanh (h j) j$;

$(2) \mathcal{X}_{\geq 3}^{+}:=\mathcal{X}_{\geq 3}^{+}(\eta, \psi, z, \bar{z})$ satisfies $\left\|\mathcal{X}_{\geq 3}^{+}\right\|_{\dot{H}^{s-\frac{3}{2}}} \leq C(s)\|z\|_{\dot{H}^{s}}^{3}$ and the "energy estimate"

$$
\operatorname{Re} \int_{\mathbb{T}}|D|^{s} \mathcal{X}_{\geq 3}^{+} \cdot \overline{|D|^{s} z} d x \leq C(s)\|z\|_{\dot{H}^{s}}^{4} .
$$

The main point of Theorem 1.1 is the construction of the bounded and invertible transformation $\mathfrak{B}(\eta, \psi)$ in (1.9) which recasts (1.1) in the Birkhoff normal form (1.10), where the cubic vector field satisfies the energy estimate (1.13). We remark that Craig-Sulem [14] constructed a bounded and symplectic transformation that conjugates (1.1) to its cubic Birkhoff normal form, but the cubic terms of the transformed vector field do not satisfy energy estimates.

We underline that, for general values of gravity, surface tension and depth $(g, \kappa, h)$, the "resonant" Birkhoff normal form Hamiltonian $H_{B N F}^{(3)}$ in (1.11) is non zero, because the system

$$
\sigma_{1} \Omega\left(j_{1}\right)+\sigma_{2} \Omega\left(j_{2}\right)+\sigma_{3} \Omega\left(j_{3}\right)=0, \quad \sigma_{1} j_{1}+\sigma_{2} j_{2}+\sigma_{3} j_{3}=0,
$$

for $\sigma_{j}= \pm$, may possess integer solutions $j_{1}, j_{2}, j_{3} \neq 0$, known as 3-waves resonances (cases with absence of 3-waves resonances are discussed in remark 4.5). The resonant Hamiltonian $H_{B N F}^{(3)}$ gives rise to a complicated dynamics, which, in fluid mechanics, is responsible for the phenomenon of the Wilton ripples. Nevertheless we are able to prove the following long time stability result. 
Theorem 1.2. (Quadratic life span) For any value of $(\kappa, g, h), \kappa>0, g \geq 0, h \in(0,+\infty]$, there exists $s_{0}>0$ and, for all $s \geq s_{0}$, there are $\varepsilon_{0}>0, c>0, C>0$, such that, for any $0<\varepsilon \leq \varepsilon_{0}$, any initial data

$$
\left(\eta_{0}, \psi_{0}\right) \in H_{0}^{s+\frac{1}{4}}(\mathbb{T}, \mathbb{R}) \times \dot{H}^{s-\frac{1}{4}}(\mathbb{T}, \mathbb{R}) \quad \text { with } \quad\left\|\eta_{0}\right\|_{H_{0}^{s+\frac{1}{4}}}+\left\|\psi_{0}\right\|_{\dot{H}^{s-\frac{1}{4}}} \leq \varepsilon,
$$

there exists a unique classical solution $(\eta, \psi)$ of (1.1) belonging to

$$
C^{0}\left(\left[-T_{\varepsilon}, T_{\varepsilon}\right], H_{0}^{s+\frac{1}{4}}(\mathbb{T}, \mathbb{R}) \times \dot{H}^{s-\frac{1}{4}}(\mathbb{T}, \mathbb{R})\right) \quad \text { with } \quad T_{\varepsilon} \geq c \varepsilon^{-2},
$$

satisfying $(\eta, \psi)_{\left.\right|_{t=0}}=\left(\eta_{0}, \psi_{0}\right)$. Moreover

$$
\sup _{t \in\left[-T_{\varepsilon}, T_{\varepsilon}\right]}\left(\|\eta\|_{H_{0}^{s+\frac{1}{4}}}+\|\psi\|_{\dot{H}^{s-\frac{1}{4}}}\right) \leq C \varepsilon .
$$

Before presenting the literature about $\varepsilon^{-2}$ existence results for water waves, we describe some key points concerning the proof of these results:

1. The long time existence Theorem 1.2 is deduced by the complete conjugation of the water waves vector field (1.1) to its Birkhoff normal form up to degree 3, Theorem 1.1, and not just on the construction of modified energies.

2. Since the gravity-capillary dispersion relation $\sim|\xi|^{\frac{3}{2}}$ is superlinear, the water waves equations (1.1) can be reduced, as in [6], to a paradifferential system with constant coefficient symbols, up to smoothing remainders (see Proposition 3.2). At the beginning of Section 4 we remark that, thanks to the $x$-translation invariance of the equations, the symbols in (3.9) of the quadratic paradifferential vector fields are actually zero. For this reason, in Section 4 it just remains to perform a Poincaré- Birkhoff normal form on the quadratic smoothing vector fields, see Proposition 4.3 .

3. Despite the fact that our transformations are non-symplectic (as in [6], [7]), we prove, in Section [5.1, using a normal form identification argument (simpler than in [7]), that the quadratic Poincaré-Birkhoff normal form term in 4.9 coincides with the Hamiltonian vector field $\mathrm{i} \partial_{\bar{z}} H_{B N F}^{(3)}$ with Hamiltonian 1.11).

4. The Hamiltonian $H_{\mathbb{C}}^{(2)}(z):=\int_{\mathbb{T}} \Omega(D) z \cdot \bar{z} d x$ is a prime integral of the resonant Birkhoff normal form $\partial_{t} z=\mathrm{i} \Omega(D) z+\mathrm{i} \partial_{\bar{z}} H_{B N F}^{(3)}(z, \bar{z})$. Moreover, since (1.14) admits at most finitely many integer solutions (Lemma 4.4) the Hamiltonian $H_{B N F}^{(3)}(z, \bar{z})=H_{B N F}^{(3)}\left(z_{L}, \bar{z}_{L}\right)$ where $z_{L}:=\sum_{0<|j| \leq \mathrm{C}} z_{j} e^{\mathrm{i} j x}$, for some finite $\mathrm{C}>0$. Therefore, any solution $z(t)$ of the Birkhoff normal form satisfies, for any $s \geq 0$,

$$
\left\|z_{L}(t)\right\|_{\dot{H}^{s}}^{2} \lesssim s\left\|z_{L}(t)\right\|_{L^{2}}^{2} \lesssim H_{\mathbb{C}}^{(2)}\left(z_{L}(t)\right)=H_{\mathbb{C}}^{(2)}\left(z_{L}(0)\right), \quad \forall t \in \mathbb{R}
$$

and $\|z(t)\|_{\dot{H}^{s}}^{2}$ remains bounded for all times. Finally we deduce the energy estimate (5.27) for the solution of the whole system (1.10), where we take into account the effect of $\mathcal{X}_{\geq 3}^{+}$, which implies stability for all $|t| \leq c \varepsilon^{-2}$. 
Literature. Local existence results for the initial value problem of the water waves equations go back to the pioneering works of Nalimov [27], Yosihara [34], Craig [12] for small initial data, Wu [30, 31] without smallness assumptions, and Beyer-Günther [9] in presence of surface tension. For some recent results about gravity-capillary waves we refer to [4, 26, 11, 28, 10, 1]. Clearly, specializing these results for initial data of size $\varepsilon$, the solutions exist and stay regular for times of order $\varepsilon^{-1}$.

Global well-posedness. In the case $x \in \mathbb{R}^{d}$ and the initial data decay sufficiently fast at infinity, global in time solutions have been constructed exploiting the dispersive effects of the system. The first global in time solutions were proved in $d=2$ by Germain-Masmoudi-Shatah [17] and $\mathrm{Wu}$ [33] for gravity water waves, by Germain-Masmoudi-Shatah [18] for the capillary problem, and for gravity-capillary water waves by Deng-Ionescu-Pausader-Pusateri [15]. In $d=1$ an almost global existence result for gravity waves was proved by $\mathrm{Wu}$ [32], improved to global regularity by Ionescu-Pusateri [23], Alazard-Delort [2], Hunter and Ifrim-Tataru [20, 21]. For capillary waves, global regularity was proved by Ionescu-Pusateri [24] and Ifrim-Tataru [22].

Normal forms. For space periodic water waves in absence of 3-waves resonances, existence results for times of order $\varepsilon^{-2}$ have been obtained in [32, 29, 23, 2, 20] for $1 d$ pure gravity waves, in [24, 22] for pure capillarity waves, and in [19] for $1 d$ gravity waves over a flat bottom. If $x \in \mathbb{T}^{2}$ we refer to [25] for an $\varepsilon^{-\frac{5}{3}+}$ result. The only $\varepsilon^{-3}$ existence result for parameter independent water waves is proved in [7], and it is based on the complete integrability of the fourth order Birkhoff normal form for $1 d$ pure gravity water waves in infinite depth.

An almost global existence result of periodic gravity-capillary water waves, even in $x$, for times $O\left(\varepsilon^{-N}\right)$ has been proved by Berti-Delort [6], for almost all values of $(g, \kappa)$. The restriction on the parameters $(g, \kappa)$ arises to verify the absence of $N$-waves interactions at any $N$. The restriction to even in $x$ solutions arises because the transformations in [6] are reversibility preserving but not symplectic. Almost global existence results for fully nonlinear reversible Schrödinger equations have been proved in [16].

We finally mention that time quasi-periodic solutions for (1.1) have been constructed in Berti-Montalto [8] and, for pure gravity waves, in Baldi-Berti-Haus-Montalto [5].

Acknowledgements. The research was partially supported by PRIN 2015 KB9WPT-005 and ERC project FAnFArE, n. 637510.

\section{Functional Setting and Paradifferential calculus}

In this section we recall definitions and results of para-differential calculus following Chapter 3 of [6], where we refer for more information. In the sequel we will deal with parameters

$$
s \geq s_{0} \gg K \gg \rho \gg 1 \text {. }
$$

Given an interval $I \subset \mathbb{R}$, symmetric with respect to $t=0$, and $s \in \mathbb{R}$, we define the space $C_{*}^{K}\left(I, \dot{H}^{s}\left(\mathbb{T}, \mathbb{C}^{2}\right)\right):=\bigcap_{k=0}^{K} C^{k}\left(I ; \dot{H}^{s-\frac{3}{2} k}\left(\mathbb{T} ; \mathbb{C}^{2}\right)\right)$ endowed with the norm

$$
\sup _{t \in I}\|U(t, \cdot)\|_{K, s} \quad \text { where } \quad\|U(t, \cdot)\|_{K, s}:=\sum_{k=0}^{K}\left\|\partial_{t}^{k} U(t, \cdot)\right\|_{\dot{H}^{s-\frac{3}{2} k}} .
$$


With similar meaning we consider $C_{*}^{K}\left(I ; \dot{H}^{s}(\mathbb{T} ; \mathbb{C})\right)$. We denote by $C_{* \mathbb{R}}^{K}\left(I, \dot{H}^{s}\left(\mathbb{T}, \mathbb{C}^{2}\right)\right)$ the subspace of functions $U$ in $C_{*}^{K}\left(I, \dot{H}^{s}\left(\mathbb{T}, \mathbb{C}^{2}\right)\right)$ such that $U=\left[\frac{u}{u}\right]$. Given $r>0$ we set

$$
B_{s}^{K}(I ; r):=\left\{U \in C_{*}^{K}\left(I, \dot{H}^{s}\left(\mathbb{T} ; \mathbb{C}^{2}\right)\right): \sup _{t \in I}\|U(t, \cdot)\|_{K, s}<r\right\} .
$$

We expand a $2 \pi$-periodic function $u(x)$, with zero average in $x$, (which is identified with $u$ in the homogeneous space), in Fourier series as

$$
u(x)=\sum_{n \in \mathbb{Z} \backslash\{0\}} \widehat{u}(n) \frac{e^{\mathrm{i} n x}}{\sqrt{2 \pi}}, \quad \widehat{u}(n):=\frac{1}{\sqrt{2 \pi}} \int_{\mathbb{T}} u(x) e^{-\mathrm{i} n x} d x .
$$

We also use the notation $u_{n}^{+}:=u_{n}:=\widehat{u}(n)$ and $u_{n}^{-}:=\overline{u_{n}}:=\overline{\widehat{u}(n)}$. We set $u^{+}(x):=u(x)$ and $u^{-}(x):=\overline{u(x)}$.

For $n \in \mathbb{N}^{*}:=\mathbb{N} \backslash\{0\}$ we denote by $\Pi_{n}$ the orthogonal projector from $L^{2}(\mathbb{T} ; \mathbb{C})$ to the subspace spanned by $\left\{e^{\mathrm{i} i x}, e^{-\mathrm{i} n x}\right\}$, i.e. $\left(\Pi_{n} u\right)(x):=\widehat{u}(n) \frac{e^{\mathrm{i} n x}}{\sqrt{2 \pi}}+\widehat{u}(-n) \frac{e^{-\mathrm{i} n x}}{\sqrt{2 \pi}}$, and we denote by $\Pi_{n}$ also the corresponding projector in $L^{2}\left(\mathbb{T}, \mathbb{C}^{2}\right)$. If $\mathcal{U}=\left(U_{1}, \ldots, U_{p}\right)$ is a $p$-tuple of functions, $\vec{n}=\left(n_{1}, \ldots, n_{p}\right) \in\left(\mathbb{N}^{*}\right)^{p}$, we set $\Pi_{\vec{n}} \mathcal{U}:=\left(\Pi_{n_{1}} U_{1}, \ldots, \Pi_{n_{p}} U_{p}\right)$.

We deal with vector fields $X$ which satisfy the $x$-translation invariance property

$$
X \circ \tau_{\theta}=\tau_{\theta} \circ X, \quad \forall \theta \in \mathbb{R}, \quad \text { where } \quad \tau_{\theta}: u(x) \mapsto\left(\tau_{\theta} u\right)(x):=u(x+\theta) .
$$

Para-differential operators. We first give the definition of the classes of symbols, collecting Definitions 3.1, 3.2 and 3.4 in [6]. Roughly speaking, the class $\widetilde{\Gamma}_{p}^{m}$ contains homogeneous symbols of order $m$ and homogeneity $p$ in $U$, while the class $\Gamma_{K, K^{\prime}, p}^{m}$ contains non-homogeneous symbols of order $m$ which vanish at degree at least $p$ in $U$, and that are $\left(K-K^{\prime}\right)$-times differentiable in $t$.

Definition 2.1. (Classes of symbols) Let $m \in \mathbb{R}, p, N \in \mathbb{N}$ with $p \leq N, K, K^{\prime}$ in $\mathbb{N}$ with $K^{\prime} \leq K, r>0$.

(i) $p$-homogeneous symbols. We denote by $\widetilde{\Gamma}_{p}^{m}$ the space of symmetric p-linear maps from $\left(\dot{H}^{\infty}\left(\mathbb{T} ; \mathbb{C}^{2}\right)\right)^{p}$ to the space of $C^{\infty}$ functions of $(x, \xi) \in \mathbb{T} \times \mathbb{R}, \mathcal{U} \rightarrow((x, \xi) \rightarrow a(\mathcal{U} ; x, \xi))$, satisfying the following. There is $\mu>0$ and, for any $\alpha, \beta \in \mathbb{N}$, there is $C>0$ such that

$$
\left|\partial_{x}^{\alpha} \partial_{\xi}^{\beta} a\left(\Pi_{\vec{n}} \mathcal{U} ; x, \xi\right)\right| \leq C|\vec{n}|^{\mu+\alpha}\langle\xi\rangle^{m-\beta} \prod_{j=1}^{p}\left\|\Pi_{n_{j}} U_{j}\right\|_{L^{2}}
$$

for any $\mathcal{U}=\left(U_{1}, \ldots, U_{p}\right)$ in $\left(\dot{H}^{\infty}\left(\mathbb{T} ; \mathbb{C}^{2}\right)\right)^{p}$, and $\vec{n}=\left(n_{1}, \ldots, n_{p}\right) \in\left(\mathbb{N}^{*}\right)^{p}$. Moreover we assume that, if for some $\left(n_{0}, \ldots, n_{p}\right) \in \mathbb{N} \times\left(\mathbb{N}^{*}\right)^{p}, \Pi_{n_{0}} a\left(\Pi_{n_{1}} U_{1}, \ldots, \Pi_{n_{p}} U_{p} ; \cdot\right) \neq 0$, then there exists a choice of signs $\sigma_{0}, \ldots, \sigma_{p} \in\{-1,1\}$ such that $\sum_{j=0}^{p} \sigma_{j} n_{j}=0$. For $p=0$ we denote by $\widetilde{\Gamma}_{0}^{m}$ the space of constant coefficients symbols $\xi \mapsto a(\xi)$ which satisfy (2.3) with $\alpha=0$ and the right hand side replaced by $C\langle\xi\rangle^{m-\beta}$. In addition we require the translation invariance property

$$
a\left(\tau_{\theta} \mathcal{U} ; x, \xi\right)=a(\mathcal{U} ; x+\theta, \xi), \quad \forall \theta \in \mathbb{R} .
$$


(ii) Non-homogeneous symbols. Let $p \geq 1$. We denote by $\Gamma_{K, K^{\prime}, p}^{m}[r]$ the space of functions $(U ; t, x, \xi) \mapsto a(U ; t, x, \xi)$, defined for $U \in B_{s_{0}}^{K}(I ; r)$, for some large enough $s_{0}$, with complex values such that for any $0 \leq k \leq K-K^{\prime}$, any $\sigma \geq s_{0}$, there are $C>0,0<r(\sigma)<r$ and for any $U \in B_{s_{0}}^{K}(I ; r(\sigma)) \cap C_{*}^{k+K^{\prime}}\left(I, \dot{H}^{\sigma}\left(\mathbb{T} ; \mathbb{C}^{2}\right)\right)$ and any $\alpha, \beta \in \mathbb{N}$, with $\alpha \leq \sigma-s_{0}$

$$
\left|\partial_{t}^{k} \partial_{x}^{\alpha} \partial_{\xi}^{\beta} a(U ; t, x, \xi)\right| \leq C\langle\xi\rangle^{m-\beta}\|U\|_{k+K^{\prime}, s_{0}}^{p-1}\|U\|_{k+K^{\prime}, \sigma} .
$$

(iii) Symbols. We denote by $\Sigma \Gamma_{K, K^{\prime}, p}^{m}[r, N]$ the space of functions $(U, t, x, \xi) \rightarrow a(U ; t, x, \xi)$ such that there are homogeneous symbols $a_{q} \in \widetilde{\Gamma}_{q}^{m}, q=p, \ldots, N-1$, and a non-homogeneous symbol $a_{N} \in \Gamma_{K, K^{\prime}, N}^{m}[r]$ such that $a(U ; t, x, \xi)=\sum_{q=p}^{N-1} a_{q}(U, \ldots, U ; x, \xi)+a_{N}(U ; t, x, \xi)$. We denote by $\Sigma \Gamma_{K, K^{\prime}, p}^{m}[r, N] \otimes \mathcal{M}_{2}(\mathbb{C})$ the space $2 \times 2$ matrices with entries in $\Sigma \Gamma_{K, K^{\prime}, p}^{m}[r, N]$.

As a consequence of the momentum condition (2.4) a symbol $a_{1}$ in the class $\widetilde{\Gamma}_{1}^{m}$, for some $m \in \mathbb{R}$, can be written as

$$
a_{1}(U ; x, \xi)=\sum_{j \in \mathbb{Z} \backslash\{0\}, \sigma= \pm}\left(a_{1}\right)_{j}^{\sigma}(\xi) u_{j}^{\sigma} e^{\mathrm{i} \sigma j x}
$$

for some coefficients $\left(a_{1}\right)_{j}^{\sigma}(\xi) \in \mathbb{C}$, see [7].

Remark 2.2. A symbol $a_{1} \in \widetilde{\Gamma}_{1}^{m}$ of the form (2.6), independent of $x$, is actually $a_{1} \equiv 0$.

We also define classes of functions in analogy with our classes of symbols.

Definition 2.3. (Functions) Fix $N, p \in \mathbb{N}$ with $p \leq N, K, K^{\prime} \in \mathbb{N}$ with $K^{\prime} \leq K, r>0$. We denote by $\widetilde{\mathcal{F}}_{p}$, resp. $\mathcal{F}_{K, K^{\prime}, p}[r], \Sigma \mathcal{F}_{p}[r, N]$, the subspace of $\widetilde{\Gamma}_{p}^{0}$, resp. $\Gamma_{p}^{0}[r]$, resp. $\Sigma \Gamma_{p}^{0}[r, N]$, made of those symbols which are independent of $\xi$. We write $\widetilde{\mathcal{F}}_{p}^{\mathbb{R}}$, resp. $\mathcal{F}_{K, K^{\prime}, p}^{\mathbb{R}}[r], \Sigma \mathcal{F}_{p}^{\mathbb{R}}[r, N]$, to denote functions in $\widetilde{\mathcal{F}}_{p}$, resp. $\mathcal{F}_{K, K^{\prime}, p}[r], \Sigma \mathcal{F}_{p}[r, N]$, which are real valued.

Paradifferential quantization. Given $p \in \mathbb{N}$ we consider functions $\chi_{p} \in C^{\infty}\left(\mathbb{R}^{p} \times \mathbb{R} ; \mathbb{R}\right)$ and $\chi \in C^{\infty}(\mathbb{R} \times \mathbb{R} ; \mathbb{R})$, even with respect to each of their arguments, satisfying, for $0<\delta \ll 1$,

$$
\begin{array}{ll}
\operatorname{supp} \chi_{p} \subset\left\{\left(\xi^{\prime}, \xi\right) \in \mathbb{R}^{p} \times \mathbb{R} ;\left|\xi^{\prime}\right| \leq \delta\langle\xi\rangle\right\}, & \chi_{p}\left(\xi^{\prime}, \xi\right) \equiv 1 \text { for }\left|\xi^{\prime}\right| \leq \delta\langle\xi\rangle / 2, \\
\operatorname{supp} \chi \subset\left\{\left(\xi^{\prime}, \xi\right) \in \mathbb{R} \times \mathbb{R} ;\left|\xi^{\prime}\right| \leq \delta\langle\xi\rangle\right\}, & \chi\left(\xi^{\prime}, \xi\right) \equiv 1 \text { for }\left|\xi^{\prime}\right| \leq \delta\langle\xi\rangle / 2 .
\end{array}
$$

For $p=0$ we set $\chi_{0} \equiv 1$. We assume moreover that $\left|\partial_{\xi}^{\alpha} \partial_{\xi^{\prime}}^{\beta} \chi_{p}\left(\xi^{\prime}, \xi\right)\right| \leq C_{\alpha, \beta}\langle\xi\rangle^{-\alpha-|\beta|}$, $\forall \alpha \in \mathbb{N}, \beta \in \mathbb{N}^{p}$, and $\left|\partial_{\xi}^{\alpha} \partial_{\xi^{\prime}}^{\beta} \chi\left(\xi^{\prime}, \xi\right)\right| \leq C_{\alpha, \beta}\langle\xi\rangle^{-\alpha-\beta}, \forall \alpha, \beta \in \mathbb{N}$.

If $a(x, \xi)$ is a smooth symbol we define its Weyl quantization as the operator acting on a $2 \pi$-periodic function $u(x)$ (written as in (2.2)) as

$$
O p^{W}(a) u=\frac{1}{\sqrt{2 \pi}} \sum_{k \in \mathbb{Z}}\left(\sum_{j \in \mathbb{Z}} \widehat{a}\left(k-j, \frac{k+j}{2}\right) \widehat{u}(j)\right) \frac{e^{\mathrm{i} k x}}{\sqrt{2 \pi}}
$$

where $\widehat{a}(k, \xi)$ is the $k^{t h}$-Fourier coefficient of the $2 \pi$-periodic function $x \mapsto a(x, \xi)$. 
Definition 2.4. (Bony-Weyl quantization) If a is a symbol in $\widetilde{\Gamma}_{p}^{m}$, respectively in $\Gamma_{K, K^{\prime}, p}^{m}{ }^{[r]}$, we set

$$
\begin{aligned}
& a_{\chi_{p}}(\mathcal{U} ; x, \xi):=\sum_{\vec{n} \in \mathbb{N}^{p}} \chi_{p}(\vec{n}, \xi) a\left(\Pi_{\vec{n}} \mathcal{U} ; x, \xi\right), \\
& a_{\chi}(U ; t, x, \xi):=\frac{1}{2 \pi} \int_{\mathbb{T}} \chi\left(\xi^{\prime}, \xi\right) \widehat{a}\left(U ; t, \xi^{\prime}, \xi\right) e^{\mathrm{i} \xi^{\prime} x} d \xi^{\prime},
\end{aligned}
$$

where in the last equality $\widehat{a}$ stands for the Fourier transform with respect to the $x$ variable, and we define the Bony-Weyl quantization of a as

$$
O p^{\mathrm{BW}}(a(\mathcal{U} ; \cdot))=O p^{W}\left(a_{\chi_{p}}(\mathcal{U} ; \cdot)\right), \quad O p^{\mathrm{BW}}(a(U ; t, \cdot))=O p^{W}\left(a_{\chi}(U ; t, \cdot)\right) .
$$

If a is a symbol in $\Sigma \Gamma_{K, K^{\prime}, p}^{m}[r, N]$, we define its Bony-Weyl quantization $O p^{\mathrm{BW}}(a(U ; t, \cdot))=$ $\sum_{q=p}^{N-1} O p^{\mathrm{BW}}\left(a_{q}(U, \ldots, U ; \cdot)\right)+O p^{\mathrm{BW}}\left(a_{N}(U ; t, \cdot)\right)$.

Paradifferential operators act on homogeneous spaces. If $a$ is in $\Sigma \Gamma_{K, K^{\prime}, p}^{m}[r, N]$, the corresponding para-differential operator is bounded from $\dot{H}^{s}$ to $\dot{H}^{s-m}$, for all $s \in \mathbb{R}$, see Proposition 3.8 in [6].

Definition 2.4 is independent of the cut-off functions $\chi_{p}, \chi$, up to smoothing operators that we define below (see Definition 3.7 in [6]). Roughly speaking, the class $\widetilde{\mathcal{R}}_{p}^{-\rho}$ contains smoothing operators which gain $\rho$ derivatives and are homogeneous of degree $p$ in $U$, while the class $\mathcal{R}_{K, K^{\prime}, p}^{-\rho}$ contains non-homogeneous $\rho$-smoothing operators which vanish at degree at least $p$ in $U$, and are $\left(K-K^{\prime}\right)$-times differentiable in $t$.

Given $\left(n_{1}, \ldots, n_{p+1}\right) \in \mathbb{N}^{p+1}$ we denote by $\max _{2}\left(n_{1}, \ldots, n_{p+1}\right)$ the second largest among the integers $n_{1}, \ldots, n_{p+1}$.

Definition 2.5. (Classes of smoothing operators) Let $N \in \mathbb{N}^{*}, K, K^{\prime} \in \mathbb{N}$ with $K^{\prime} \leq K \in \mathbb{N}$, $\rho \geq 0$ and $r>0$.

(i) $p$-homogeneous smoothing operators. We denote by $\widetilde{\mathcal{R}}_{p}^{-\rho}$ the space of $(p+1)$-linear maps $R$ from $\left(\dot{H}^{\infty}\left(\mathbb{T} ; \mathbb{C}^{2}\right)\right)^{p} \times \dot{H}^{\infty}(\mathbb{T} ; \mathbb{C})$ to $\dot{H}^{\infty}(\mathbb{T} ; \mathbb{C})$, symmetric in $\left(U_{1}, \ldots, U_{p}\right)$, of the form $\left(U_{1}, \ldots, U_{p+1}\right) \rightarrow R\left(U_{1}, \ldots, U_{p}\right) U_{p+1}$ that satisfy the following. There are $\mu \geq 0$, $C>0$ such that

$$
\left\|\Pi_{n_{0}} R\left(\Pi_{\vec{n}} \mathcal{U}\right) \Pi_{n_{p+1}} U_{p+1}\right\|_{L^{2}} \leq C \frac{\max _{2}\left(n_{1}, \ldots, n_{p+1}\right)^{\rho+\mu}}{\max \left(n_{1}, \ldots, n_{p+1}\right)^{\rho}} \prod_{j=1}^{p+1}\left\|\Pi_{n_{j}} U_{j}\right\|_{L^{2}}
$$

for any $\mathcal{U}=\left(U_{1}, \ldots, U_{p}\right) \in\left(\dot{H}^{\infty}\left(\mathbb{T} ; \mathbb{C}^{2}\right)\right)^{p}, U_{p+1} \in \dot{H}^{\infty}(\mathbb{T} ; \mathbb{C}), \vec{n}=\left(n_{1}, \ldots, n_{p}\right) \in\left(\mathbb{N}^{*}\right)^{p}$, any $n_{0}, n_{p+1} \in \mathbb{N}^{*}$. Moreover, if

$$
\Pi_{n_{0}} R\left(\Pi_{n_{1}} U_{1}, \ldots, \Pi_{n_{p}} U_{p}\right) \Pi_{n_{p+1}} U_{p+1} \neq 0,
$$

then there is a choice of signs $\sigma_{0}, \ldots, \sigma_{p+1} \in\{ \pm 1\}$ such that $\sum_{j=0}^{p+1} \sigma_{j} n_{j}=0$. In addition we require the translation invariance property

$$
R\left(\tau_{\theta} \mathcal{U}\right)\left[\tau_{\theta} U_{p+1}\right]=\tau_{\theta}\left(R(\mathcal{U}) U_{p+1}\right), \quad \forall \theta \in \mathbb{R} .
$$


(ii) Non-homogeneous smoothing operators. We denote by $\mathcal{R}_{K, K^{\prime}, N}^{-\rho}[r]$ the space of maps $(V, U) \mapsto R(V) U$ defined on $B_{s_{0}}^{K}(I ; r) \times C_{*}^{K}\left(I, \dot{H}^{s_{0}}(\mathbb{T}, \mathbb{C})\right)$ which are linear in the variable $U$ and such that the following holds true. For any $s \geq s_{0}$ there are $C>0$ and $\left.r(s) \in\right] 0, r[$ such that, for any $V \in B_{s_{0}}^{K}(I ; r) \cap C_{*}^{K}\left(I, \dot{H}^{s}\left(\mathbb{T}, \mathbb{C}^{2}\right)\right)$, any $U \in C_{*}^{K}\left(I, \dot{H}^{s}(\mathbb{T}, \mathbb{C})\right)$, any $0 \leq$ $k \leq K-K^{\prime}$ and any $t \in I$, we have

$$
\begin{array}{rl}
\left\|\partial_{t}^{k}(R(V) U)(t, \cdot)\right\|_{\dot{H}^{s-\frac{3}{2} k+\rho}} \leq \sum_{k^{\prime}+k^{\prime \prime}=k} & C\left(\|U\|_{k^{\prime \prime}, s}\|V\|_{k^{\prime}+K^{\prime}, s_{0}}^{N}\right. \\
& \left.+\|U\|_{k^{\prime \prime}, s_{0}}\|V\|_{k^{\prime}+K^{\prime}, s_{0}}^{N-1}\|V\|_{k^{\prime}+K^{\prime}, s}\right) .
\end{array}
$$

(iii) Smoothing operators. We denote by $\Sigma \mathcal{R}_{K, K^{\prime}, p}^{-\rho}[r, N]$ the space of maps $(V, t, U) \rightarrow$ $R(V ; t) U$ that may be written as $R(V ; t) U=\sum_{q=p}^{N-1} R_{q}(V, \ldots, V) U+R_{N}(V ; t) U$ for some $R_{q}$ in $\widetilde{\mathcal{R}}_{q}^{-\rho}, q=p, \ldots, N-1$ and $R_{N}$ in $\mathcal{R}_{K, K^{\prime}, N}^{-\rho}[r]$.

We denote by $\Sigma \mathcal{R}_{K, K^{\prime}, p}^{-\rho}[r, N] \otimes \mathcal{M}_{2}(\mathbb{C})$ the space of $2 \times 2$ matrices with entries in the class $\Sigma \mathcal{R}_{K, K^{\prime}, p}^{-\rho}[r, N]$.

Below we introduce classes of operators without keeping track of the number of lost derivatives in a precise way (see Definition 3.9 in [6]). The class $\widetilde{\mathcal{M}}_{p}^{m}$ denotes multilinear maps that lose $m$ derivatives and are $p$-homogeneous in $U$, while the class $\mathcal{M}_{K, K^{\prime}, p}^{m}$ contains non-homogeneous maps which lose $m$ derivatives, vanish at degree at least $p$ in $U$, and are $\left(K-K^{\prime}\right)$-times differentiable in $t$.

Definition 2.6. (Classes of maps) Let $p, N \in \mathbb{N}$, with $p \leq N, N \geq 1, K, K^{\prime} \in \mathbb{N}$ with $K^{\prime} \leq K$ and $m \geq 0$.

(i) $p$-homogeneous maps. We denote by $\widetilde{\mathcal{M}}_{p}^{m}$ the space of $(p+1)$-linear maps $M$ from $\left(\dot{H}^{\infty}\left(\mathbb{T} ; \mathbb{C}^{2}\right)\right)^{p} \times \dot{H}^{\infty}(\mathbb{T} ; \mathbb{C})$ to $\dot{H}^{\infty}(\mathbb{T} ; \mathbb{C})$ which are symmetric in $\left(U_{1}, \ldots, U_{p}\right)$, of the form $\left(U_{1}, \ldots, U_{p+1}\right) \rightarrow M\left(U_{1}, \ldots, U_{p}\right) U_{p+1}$ and that satisfy the following. There is $C>0$ such that

$$
\left\|\Pi_{n_{0}} M\left(\Pi_{\vec{n}} \mathcal{U}\right) \Pi_{n_{p+1}} U_{p+1}\right\|_{L^{2}} \leq C\left(n_{0}+n_{1}+\cdots+n_{p+1}\right)^{m} \prod_{j=1}^{p+1}\left\|\Pi_{n_{j}} U_{j}\right\|_{L^{2}}
$$

for any $\mathcal{U}=\left(U_{1}, \ldots, U_{p}\right) \in\left(\dot{H}^{\infty}\left(\mathbb{T} ; \mathbb{C}^{2}\right)\right)^{p}$, any $U_{p+1} \in \dot{H}^{\infty}(\mathbb{T} ; \mathbb{C}), \vec{n}=\left(n_{1}, \ldots, n_{p}\right)$ in $\left(\mathbb{N}^{*}\right)^{p}$, any $n_{0}, n_{p+1} \in \mathbb{N}^{*}$. Moreover the properties (2.8)-(2.9) hold.

(ii) Non-homogeneous maps. We denote by $\mathcal{M}_{K, K^{\prime}, N}^{m}[r]$ the space of maps $(V, u) \mapsto$ $M(V) U$ defined on $B_{s_{0}}^{K}(I ; r) \times C_{*}^{K}\left(I, \dot{H}^{s_{0}}(\mathbb{T}, \mathbb{C})\right)$ which are linear in the variable $U$ and such that the following holds true. For any $s \geq s_{0}$ there are $C>0$ and $\left.r(s) \in\right] 0, r[$ such that for any $V \in B_{s_{0}}^{K}(I ; r) \cap C_{*}^{K}\left(I, \dot{H}^{s}\left(\mathbb{T}, \mathbb{C}^{2}\right)\right)$, any $U \in C_{*}^{K}\left(I, \dot{H}^{s}(\mathbb{T}, \mathbb{C})\right)$, any $0 \leq k \leq K-K^{\prime}$, $t \in I$, we have that $\left\|\partial_{t}^{k}(M(V) U)(t, \cdot)\right\|_{\dot{H}^{s-\frac{3}{2} k-m}}$ is bounded by the right hand side of (2.10).

(iii) Maps. We denote by $\Sigma \mathcal{M}_{K, K^{\prime}, p}^{m}[r, N]$ the space of maps $(V, t, U) \rightarrow M(V ; t) U$ that may be written as $M(V ; t) U=\sum_{q=p}^{N-1} M_{q}(V, \ldots, V) U+M_{N}(V ; t) U$ for some $M_{q}$ 
in $\widetilde{\mathcal{M}}_{q}^{m}, q=p, \ldots, N-1$ and $M_{N}$ in $\mathcal{M}_{K, K^{\prime}, N}^{m}[r]$. Finally we set $\widetilde{\mathcal{M}}_{p}:=\cup_{m \geq 0} \widetilde{\mathcal{M}}_{p}^{m}$, $\mathcal{M}_{K, K^{\prime}, p}[r]:=\cup_{m \geq 0} \mathcal{M}_{K, K^{\prime}, p}^{m}[r], \Sigma \mathcal{M}_{K, K^{\prime}, p}[r, N]:=\cup_{m \geq 0} \Sigma \mathcal{M}_{K, K^{\prime}, p}^{m}[r]$.

We denote by $\Sigma \mathcal{M}_{K, K^{\prime}, p}^{m}[r, N] \otimes \mathcal{M}_{2}(\mathbb{C})$ the space of $2 \times 2$ matrices whose entries are maps in $\Sigma \mathcal{M}_{K, K^{\prime}, p}^{m}[r, N]$. We set $\Sigma \mathcal{M}_{K, K^{\prime}, p}[r, N] \otimes \mathcal{M}_{2}(\mathbb{C}):=\cup_{m \in \mathbb{R}} \Sigma \mathcal{M}_{K, K^{\prime}, p}^{m}[r, N] \otimes \mathcal{M}_{2}(\mathbb{C})$.

Given an operator $\mathrm{R}_{1}$ in $\widetilde{\mathcal{R}}_{1}^{-\rho}$ (or in $\widetilde{\mathcal{M}}_{1}^{m}$ ), and $z^{\sigma_{2}}, \sigma_{2}= \pm$, the momentum condition (2.9) implies that

$$
\mathrm{R}_{1}(U)\left[z^{\sigma_{2}}\right]=\sum_{j_{1}, j_{2} \in \mathbb{Z} \backslash\{0\}, \sigma_{1}= \pm}\left(\mathrm{R}_{1}\right)_{j_{1}, j_{2}}^{\sigma_{1}, \sigma_{2}} u_{j_{1}}^{\sigma_{1}} z_{j_{2}}^{\sigma_{2}} e^{\mathrm{i}\left(\sigma_{1} j_{1}+\sigma_{2} j_{2}\right) x}
$$

for some $\left(\mathrm{R}_{1}\right)_{j_{1}, j_{2}}^{\sigma_{1}, \sigma_{2}} \in \mathbb{C}$, see [7].

Proposition 2.7. (Compositions) Let $m, m^{\prime} \in \mathbb{R}, N, K, K^{\prime} \in \mathbb{N}$ with $K^{\prime} \leq K, p_{1}, p_{2}, p_{3} \in \mathbb{N}$, $\rho \geq 0$ and $r>0$. Let $a \in \Sigma \Gamma_{K, K^{\prime}, p_{1}}^{m}[r, N], R \in \Sigma \mathcal{R}_{K, K^{\prime}, p_{2}}^{-\rho}[r, N]$ and $M \in \Sigma \mathcal{M}_{K, K^{\prime}, p_{3}}^{m^{\prime}}[r, N]$. Then:

(i) $R(U ; t) \circ O p^{\mathrm{BW}}(a(U ; t, x, \xi)), O p^{\mathrm{BW}}(a(U ; t, x, \xi)) \circ R(U ; t)$ are in $\Sigma \mathcal{R}_{K, K^{\prime}, p_{1}+p_{2}}^{-\rho+m}[r, N]$;

(ii) $R(U ; t) \circ M(U ; t)$ and $M(U ; t) \circ R(U ; t)$ are smoothing operators in $\Sigma \mathcal{R}_{K, K^{\prime}, p_{2}+p_{3}}^{-\rho+m^{\prime}}[r, N]$;

(iii) If $R_{1} \in \widetilde{\mathcal{R}}_{p_{1}}^{-\rho}, p_{1} \geq 1$, then $R_{1}(\underbrace{U, \ldots, U}_{p_{1}-1}, M(U ; t) U)$ belongs to $\Sigma \mathcal{R}_{K, K^{\prime}, p_{1}+p_{3}}^{-\rho+m^{\prime}}[r, N]$.

Proof. See Propositions 3.16, 3.17 in [6]. The translation invariance properties for the composed operators and symbols in items (i)-(ii) follow as in [7].

Real-to-real operators. Given a linear operator $R(U)[\cdot]$ acting on $\mathbb{C}$ (it may be a smoothing operator in $\Sigma \mathcal{R}_{K, K^{\prime}, 1}^{-\rho}$ or a map in $\left.\Sigma \mathcal{M}_{K, K^{\prime}, 1}\right)$ we associate the linear operator defined by

$$
\bar{R}(U)[v]:=\overline{R(U)[\bar{v}]}, \quad \forall v \in \mathbb{C} .
$$

We say that a matrix of operators acting on $\mathbb{C}^{2}$ is real-to-real, if it has the form

$$
R(U)=\left(\begin{array}{ll}
R_{1}(U) & R_{2}(U) \\
R_{2}(U) & \overline{R_{1}}(U)
\end{array}\right) .
$$

If $R(U)$ is a real-to-real matrix of operators then, given $V=\left[\frac{v}{v}\right]$, the vector $Z:=R(U)[V]$ has the form $Z=\left[\frac{z}{z}\right]$, i.e. the second component is the complex conjugated of the first one.

Given two linear operators $A, B$ (either two operator-valued matrices acting on $\mathbb{C}^{2}$ as in (2.12), we denote their commutator by $[A, B]=A B-B A$.

- The notation $A \lesssim_{s} B$ means that $A \leq C(s) B$ for some positive constant $C(s)>0$.

\section{Paradifferential reduction to constant symbols up to smoothing operators}

The first step in order to prove Theorem 1.1 is to write 1.1 in paradifferential form, to symmetrize it, and reduce to paradifferential symbols which are constant in $x$, see Proposition 3.2 
These results are proved in [6] (up to minor details). We denote the horizontal and vertical components of the velocity field at the free interface by

$$
\begin{aligned}
& V=V(\eta, \psi):=\left(\partial_{x} \Phi\right)(x, \eta(x))=\psi_{x}-\eta_{x} B, \\
& B=B(\eta, \psi):=\left(\partial_{y} \Phi\right)(x, \eta(x))=\frac{G(\eta) \psi+\eta_{x} \psi_{x}}{1+\eta_{x}^{2}},
\end{aligned}
$$

and the "good unknown" of Alinhac

$$
\omega:=\psi-O p^{\mathrm{BW}}(B(\eta, \psi)) \eta,
$$

as introduced in Alazard-Metivier [3]. The function $B(\eta, \psi)$ belongs to $\Sigma \mathcal{F}_{K, 0,1}^{\mathbb{R}}[r, N]$, for any $N>0$ (see Proposition 7.4 in [6]). Then, by the action of a paraproduct, if $\eta \in H_{0}^{s+\frac{1}{4}}$ and $\psi \in \dot{H}^{s-\frac{1}{4}}$ then the good unknown $\omega$ is in $\dot{H}^{s-\frac{1}{4}}$.

Define the Fourier multiplier $\Lambda$ of order $-1 / 4$ as

$$
\Lambda:=\Lambda(D):=(D \tanh (h D))^{\frac{1}{4}}\left(g+\kappa D^{2}\right)^{-\frac{1}{4}}
$$

and consider the complex function

$$
u:=\frac{1}{\sqrt{2}} \Lambda \omega+\frac{\mathrm{i}}{\sqrt{2}} \Lambda^{-1} \eta, \quad \eta=\frac{1}{\mathrm{i} \sqrt{2}} \Lambda(u-\bar{u}), \quad \omega=\frac{1}{\sqrt{2}} \Lambda^{-1}(u+\bar{u})
$$

where $\Lambda^{-1}$ acts on functions modulo constants in itself.

Let $K \in \mathbb{N}$. We first remark that, if $(\eta, \psi)$ solves the gravity-capillary system 1.11, then the function $u$ defined in (3.3) satisfies, by Proposition 7.9 in [6], for $s \gg K$, as long as $u$ stays in the unit ball of $\dot{H}^{s}(\mathbb{T}, \mathbb{C})$,

$$
\left\|\partial_{t}^{k} u\right\|_{\dot{H}^{s-\frac{3}{2} k}} \lesssim_{s, K}\|u\|_{\dot{H}^{s}}, \quad \forall 0 \leq k \leq K .
$$

As a consequence, if (1.8) holds then

$$
\sup _{t \in[-T, T]}\left\|\partial_{t}^{k} u\right\|_{\dot{H}^{s-\frac{3}{2} k}} \leq C_{s, K} \bar{\varepsilon}, \quad \forall 0 \leq k \leq K .
$$

Proposition 3.1. (Paradifferential complex form of the water waves equations) Let $N, K \in$ $\mathbb{N}^{*}, \rho>0$. Assume that $(\eta, \psi)$ solves the gravity-capillary system (1.1) and satisfy (1.8) for some $T>0$ and $s \gg K$. Then the function $U:=\left[\frac{u}{u}\right]$, with $u$ defined in (3.3), solves

$$
D_{t} U=\Omega(D) E U+O p^{\mathrm{BW}}(A(U ; t, x, \xi)) U+R(U ; t) U, E:=\left[\begin{array}{cc}
1 & 0 \\
0 & -1
\end{array}\right],
$$

where $D_{t}:=\frac{1}{\mathrm{i}} \partial_{t}$ and

- $\Omega(D)=O p^{\mathrm{BW}}(\Omega(\xi))$ where $\Omega(\xi) \in \widetilde{\Gamma}_{0}^{\frac{3}{2}}$ is the dispersion relation symbol defined in (1.5).

- the matrix of symbols $A(U ; t, x, \xi) \in \Sigma \Gamma_{K, 1,1}^{1}[r, N] \otimes \mathcal{M}_{2}(\mathbb{C})$ has the form

$$
\begin{aligned}
A(U ; t, x, \xi) & =\left(\zeta(U ; t, x) \Omega(\xi)+\lambda_{\frac{1}{2}}(U ; t, x, \xi)\right)\left[\begin{array}{cc}
1 & 0 \\
0 & -1
\end{array}\right] \\
& +\left(\zeta(U ; t, x) \Omega(\xi)+\lambda_{-\frac{1}{2}}(U ; t, x, \xi)\right)\left[\begin{array}{cc}
0 & -1 \\
1 & 0
\end{array}\right] \\
& +\lambda_{1}(U ; t, x, \xi)\left[\begin{array}{ll}
1 & 0 \\
0 & 1
\end{array}\right]+\lambda_{0}(U ; t, x, \xi)\left[\begin{array}{cc}
0 & 1 \\
1 & 0
\end{array}\right]
\end{aligned}
$$


where

- the function $\zeta(U ; t, x)$ is in $\Sigma \mathcal{F}_{K, 0,1}^{\mathbb{R}}[r, N]$;

- the symbols $\lambda_{j}(U ; t, x, \xi)$ are in $\Sigma \Gamma_{K, 1,1}^{j}[r, N], j=1,0,1 / 2,-1 / 2$, and $\operatorname{Im} \lambda_{j}(U ; t, x, \xi)$ are in $\Sigma \Gamma_{K, 1,1}^{j-1}[r, N]$ for $j=1,1 / 2$;

- the matrix of smoothing operators $R(U ; t)$ is in $\Sigma \mathcal{R}_{K, 1,1}^{-\rho}[r, N] \otimes \mathcal{M}_{2}(\mathbb{C})$;

- the operators $\mathrm{i} O p^{\mathrm{BW}}(A(U ; t, x, \xi))$ and $\mathrm{i} R(U ; t)$ are real-to-real, according to (2.12).

Proof. It is Corollary 7.7 and Proposition 7.8 in [6]. The only difference is that $U(x)$ is not even in $x$. The property that the homogeneous components $A_{p}(U ; t, x, \xi), R_{p}(U ; t), p=1, \ldots, N$, of the matrices $A(U ; t, x, \xi), R(U ; t)$ satisfy (2.4) and (2.9) is checked as in [7].

System (3.6) has the form

$$
D_{t} U=\Omega(D) E U+M(U ; t) U
$$

where $M(U ; t)$ is a real-to-real map in $\Sigma \mathcal{M}_{K, 1,1}^{m_{1}}[r, N] \otimes \mathcal{M}_{2}(\mathbb{C})$ for some $m_{1} \geq 3 / 2$ (using that paradifferential operators and smoothing remainders are maps, see (4.2.6) in [6]).

As in [6], since the dispersion law (1.5) is super-linear, system (3.6) can be transformed into a paradifferential diagonal system with a symbol constant in $x$, up to smoothing terms.

Proposition 3.2. (Reduction to constant coefficients up to smoothing operators) Fix $\rho>0$ arbitrary. There exist $s_{0}>0, K^{\prime}:=K^{\prime}(\rho)$ such that, for any $s \geq s_{0}$, for all $0<r \leq r_{0}(s)$ small enough, for all $K \geq K^{\prime}$ and any solution $U \in B_{s}^{K}(I ; r)$ of (3.6), there is a family of real-to-real, bounded, invertible linear maps $\mathfrak{F}^{\theta}(U), \theta \in[0,1]$, such that the function

$$
Z:=\left[\frac{z}{z}\right]=\left(\mathfrak{F}^{\theta}(U)\right)_{\mid \theta=1}[U]
$$

solves the system

$$
D_{t} Z=O p^{\mathrm{BW}}((1+\underline{\zeta}(U ; t)) \Omega(\xi) E+H(U ; t, \xi)) Z+R(U ; t)[Z]
$$

where

- the function $\underline{\zeta}(U ; t) \in \Sigma \mathcal{F}_{K, K^{\prime}, 1}^{\mathbb{R}}[r, N]$ and the diagonal matrix of symbols $H(U ; t, \xi) \in$ $\Sigma \Gamma_{K, K^{\prime}, 1}^{1}[r, N] \otimes \mathcal{M}_{2}(\mathbb{C})$ are independent of $x$;

- the symbol $\operatorname{Im} H(U ; t, \xi)$ belongs to $\Sigma \Gamma_{K, K^{\prime}, 1}^{0}[r, N] \otimes \mathcal{M}_{2}(\mathbb{C})$;

- $R(U ; t)$ is matrix of smoothing operators in $\Sigma \mathcal{R}_{K, K^{\prime}, 1}^{-\rho}[r, N] \otimes \mathcal{M}_{2}(\mathbb{C})$

- the operators $\mathrm{i} O p^{\mathrm{BW}}(H(U ; t, \xi))$ and $\mathrm{i} R(U ; t)$ are real-to-real, according to 2.12 ;

- the map $\mathfrak{F}^{\theta}(U)$ satisfies, for all $0 \leq k \leq K-K^{\prime}$, for any $V \in C_{* \mathbb{R}}^{K-K^{\prime}}\left(I ; \dot{H}^{s}\left(\mathbb{T} ; \mathbb{C}^{2}\right)\right)$,

$$
\left\|\partial_{t}^{k} \mathfrak{F}^{\theta}(U)[V]\right\|_{\dot{H}^{s-\frac{3}{2} k}}+\left\|\partial_{t}^{k}\left(\mathfrak{F}^{\theta}(U)\right)^{-1}[V]\right\|_{\dot{H}^{s-\frac{3}{2} k}} \leq\|V\|_{k, s}\left(1+C_{s, r, K}\|U\|_{K, s_{0}}\right)
$$

uniformly in $\theta \in[0,1]$. Moreover the map $\mathfrak{F}^{\theta}(U)=U+\theta M_{1}(U)[U]+M_{\geq 2}(\theta ; U)[U]$ where $M_{1}(U)$ is in $\widetilde{\mathcal{M}}_{1} \otimes \mathcal{M}_{2}(\mathbb{C})$ and $M_{\geq 2}(\theta ; U) \in \mathcal{M}_{K, K^{\prime}, 2}[r] \otimes \mathcal{M}_{2}(\mathbb{C})$ with estimates uniform in $\theta \in[0,1]$. 
Proof. This statement collects the results of Propositions 4.9, 5.1 and 5.5 in [6]. The remainder in (5.2.9) in [6] has the form [3.9] expressing $U=\left(\mathfrak{F}^{\theta}(U)\right)_{\mid \theta=1}^{-1} Z$ and using the estimates (3.10), which follow by Lemma 3.22 in [6]. Another difference is that $Z(x)$ is not even in $x$. The $x$-invariance properties (2.4) for the symbols and (2.9) for the smoothing operators are checked as in [7]. The last statement follows using Lemma A.2 in [7].

\section{Poincaré - Birkhoff normal form at quadratic degree}

From this section the analysis strongly differs from [6].

- Notation: for simplicity in the sequel we omit to write the dependence on the time $t$ in the symbols, smoothing remainders and maps, writing $a(U ; x, \xi), R(U), M(U)$ instead of $a(U ; t, x, \xi), R(U ; t), M(U ; t)$.

The aim of this section is to transform system (3.9) into its quadratic Poincaré-Birkhoff normal form, see system (4.9). We first observe that the paradifferential vector field in 3.9) of quadratic homogeneity is actually zero.

Lemma 4.1. (Quadratic Poincaré-Birkhoff normal form up to smoothing vector fields) The system (3.9) with $N=2$ has the form

$$
\partial_{t} Z=\mathrm{i} \Omega(D) E Z+\mathrm{R}_{1}(U)[Z]+\widetilde{\mathcal{X}}_{\geq 3}(U, Z)
$$

where $\mathrm{R}_{1}(U) \in \widetilde{\mathcal{R}}_{1}^{-\rho} \otimes \mathcal{M}_{2}(\mathbb{C})$ and

$$
\widetilde{\mathcal{X}}_{\geq 3}(U, Z)=\mathrm{i} O p^{\mathrm{BW}}\left(\mathcal{H}_{\geq 2}(U ; \xi)\right) Z+\mathrm{R}_{\geq 2}(U)[Z]
$$

where $\mathcal{H}_{\geq 2}(U ; \xi) \in \Gamma_{K, K^{\prime}, 2}^{3 / 2}[r] \otimes \mathcal{M}_{2}(\mathbb{C})$ is a diagonal matrix of symbols independent of $x$, such that

$$
\operatorname{Im} \mathcal{H}_{\geq 2}(U ; \xi) \in \Gamma_{K, K^{\prime}, 2}^{0}[r] \otimes \mathcal{M}_{2}(\mathbb{C}),
$$

and $\mathrm{R}_{\geq 2}(U) \in \mathcal{R}_{K, K^{\prime}, 2}^{-\rho}[r] \otimes \mathcal{M}_{2}(\mathbb{C})$. The operators $\mathrm{R}_{1}(U)$ and $\widetilde{\mathcal{X}}_{\geq 3}(U, Z)$ are real-to-real.

Proof. We expand in homogeneity the function $\underline{\zeta}(U)=\zeta_{1}(U)+\zeta_{\geq 2}(U), \zeta_{1} \in \widetilde{\mathcal{F}}_{1}^{\mathbb{R}}$, the diagonal matrix of symbols $H(U ; \xi)=H_{1}(U ; \xi)+\bar{H}_{\geq 2}(U ; \xi), H_{1}(U ; \xi) \in \widetilde{\Gamma}_{1}^{1} \otimes \mathcal{M}_{2}(\mathbb{C})$, and the smoothing remainder $R(U)=-\mathrm{iR}_{1}(U)-\mathrm{iR}_{\geq 2}(U), \mathrm{R}_{1}(U) \in \widetilde{\mathcal{R}}_{1}^{-\rho} \otimes \mathcal{M}_{2}(\mathbb{C})$. Since the function $\zeta_{1}(U)$ and $H_{1}(U ; \xi)$ admit an expansion as $(2.6)$ and are independent of $x$ (see Proposition 3.2), Remark 2.2 implies that $\zeta_{1}(U)=0, H_{1}(U ; \xi)=0$. This proves (4.1)-(4.3).

System (4.1) is yet in Poincaré-Birkhoff normal form at degree 2 up to smoothing remainders and the cubic term $\widetilde{\mathcal{X}}_{\geq 3}$ in (4.2) admits an energy estimate as (1.13), since $\mathcal{H}_{\geq 2}(U ; \xi)$ is independent of $x$ and purely imaginary up to symbols of order 0 , see 4.3. 
The goal is now to transform the quadratic smoothing term $\mathrm{R}_{1}(U)[Z]$ in (4.1) to PoincaréBirkhoff normal form at degree 2, see Definition 4.2 The remainder $\mathrm{R}_{1}(U)$ in (4.1) is realto-real (i.e. has the form (2.12), satisfies the momentum condition (2.9), thus it has the form (2.11), and so we write it as

$$
\mathrm{R}_{1}(U)=\left(\begin{array}{ll}
\left(\mathrm{R}_{1}(U)\right)_{+}^{+} & \left(\mathrm{R}_{1}(U)\right)_{+}^{-} \\
\left(\mathrm{R}_{1}(U)\right)_{-}^{+} & \left(\mathrm{R}_{1}(U)\right)_{-}^{-}
\end{array}\right),\left(\mathrm{R}_{1}(U)\right)_{\sigma}^{\sigma^{\prime}} \in \widetilde{\mathcal{R}}_{1}^{-\rho},\left(\mathrm{R}_{1}(U)\right)_{\sigma}^{\sigma^{\prime}}=\overline{\left(\mathrm{R}_{1}(U)\right)_{-\sigma}^{-\sigma^{\prime}}},
$$

for $\sigma, \sigma^{\prime}= \pm$. For any $\sigma, \sigma^{\prime}= \pm$ we expand

$$
\left(\mathrm{R}_{1}(U)\right)_{\sigma}^{\sigma^{\prime}}=\sum_{\epsilon= \pm}\left(\mathrm{R}_{1, \epsilon}(U)\right)_{\sigma}^{\sigma^{\prime}}
$$

where, for $\epsilon= \pm$, and $\left(\mathrm{R}_{1, \epsilon}(U)\right)_{\sigma}^{\sigma^{\prime}} \in \widetilde{\mathcal{R}}_{1}^{-\rho}$ is the homogeneous smoothing operator

$$
\left(\mathrm{R}_{1, \epsilon}(U)\right)_{\sigma}^{\sigma^{\prime}} z^{\sigma^{\prime}}=\frac{1}{\sqrt{2 \pi}} \sum_{j \in \mathbb{Z} \backslash\{0\}}\left(\sum_{k \in \mathbb{Z} \backslash\{0\}}\left(\mathrm{R}_{1, \epsilon}(U)\right)_{\sigma, j}^{\sigma^{\prime}, k} z_{k}^{\sigma^{\prime}}\right) e^{\mathrm{i} \sigma j x}
$$

with entries

$$
\left(\mathrm{R}_{1, \epsilon}(U)\right)_{\sigma, j}^{\sigma^{\prime}, k}:=\frac{1}{\sqrt{2 \pi}} \sum_{\substack{n \in \mathbb{Z} \backslash\{0\} \\ \epsilon n+\sigma^{\prime} k=\sigma j}}\left(\mathrm{r}_{1, \epsilon}\right)_{n, k}^{\sigma, \sigma^{\prime}} u_{n}^{\epsilon}, \quad j, k \in \mathbb{Z} \backslash\{0\},
$$

for suitable scalar coefficients $\left(\mathrm{r}_{1, \epsilon}\right)_{n, k}^{\sigma, \sigma^{\prime}} \in \mathbb{C}$. The restriction $\epsilon n+\sigma^{\prime} k=\sigma j$ is due to the momentum condition.

Definition 4.2. (Poincaré-Birkhoff Resonant smoothing operator) Given a real-to-real, smoothing operator $\mathrm{R}_{1}(U) \in \widetilde{\mathcal{R}}_{1}^{-\rho} \otimes \mathcal{M}_{2}(\mathbb{C})$ as in (4.4)-(4.7), we define the Poincaré-Birkhoff resonant, real-to-real, smoothing operator $R_{1}^{\text {res }}(U) \in \widetilde{\mathcal{R}}_{1}^{-\rho} \otimes \mathcal{M}_{2}(\mathbb{C})$ with matrix entries $\left(\mathrm{R}_{1, \epsilon}^{r e s}(U)\right)_{\sigma, j}^{\sigma^{\prime}, k}$ defined as in (4.7) such that, for any $\epsilon, \sigma, \sigma^{\prime}= \pm, j, k \in \mathbb{Z} \backslash\{0\}$,

$$
\left(\mathrm{R}_{1, \epsilon}^{r e s}(U)\right)_{\sigma, j}^{\sigma^{\prime}, k}=\frac{1}{\sqrt{2 \pi}} \sum_{\substack{n \in \mathbb{Z} \backslash\{0\}, \epsilon n+\sigma^{\prime} k=\sigma j \\ \sigma \Omega(j)-\sigma^{\prime} \Omega(k)-\epsilon \Omega(n)=0}}\left(\mathrm{r}_{1, \epsilon}\right)_{n, k}^{\sigma, \sigma^{\prime}} u_{n}^{\epsilon} .
$$

In the next Proposition we conjugate (4.1) into its complete quadratic Poincaré-Birkhoff normal form.

Proposition 4.3. (Quadratic Poincaré-Birkhoff normal form) There exists $\rho_{0}>0$ such that, for all $\rho \geq \rho_{0}, K \geq K^{\prime}$ with $K^{\prime}:=K^{\prime}(\rho)$ given by Proposition 3.2 there exists $s_{0}>0$ such that, for any $s \geq s_{0}$, for all $0<r \leq r_{0}(s)$ small enough, and any solution $U \in B_{s}^{K}(I ; r)$ of the water waves system (3.6), there is a family of real-to-real, bounded, invertible linear maps $\mathfrak{C}^{\theta}(U), \theta \in[0,1]$, such that, if $Z$ solves (4.1), then the function

$$
Y:=\left[\frac{y}{y}\right]=\left(\mathfrak{C}^{\theta}(U)[Z]\right)_{\mid \theta=1}
$$


solves

$$
\partial_{t} Y=\mathrm{i} \Omega(D) E Y+\mathrm{R}_{1}^{r e s}(Y)[Y]+\mathcal{X}_{\geq 3}(U, Y)
$$

where:

- $E$ is the matrix in (3.6) and $\Omega(D)$ has symbol (1.5);

- $\mathrm{R}_{1}^{\text {res }}(Y) \in \widetilde{\mathcal{R}}_{1}^{-\left(\rho-\rho_{0}\right)} \otimes \mathcal{M}_{2}(\mathbb{C})$ is the real-to-real Poincaré-Birkhoff resonant smoothing operator introduced in Definition 4.2.

- $\mathcal{X}_{\geq 3}(U, Y)$ has the form

$$
\mathcal{X}_{\geq 3}(U, Y)=\left[\frac{\mathcal{X}_{\geq 3}^{+}(U, Y)}{\mathcal{X}_{\geq 3}^{+}(U, Y)}\right]:=\mathrm{iO} p^{\mathrm{BW}}\left(\mathcal{H}_{\geq 2}(U ; \xi)\right)[Y]+\mathfrak{R}_{\geq 2}(U)[Y]
$$

where $\mathcal{H}_{\geq 2}(U ; \xi)$ is defined in (4.2) and satisfies (4.3), while $\mathfrak{R}_{\geq 2}(U)$ is a matrix of real-to-real smoothing operators in $\mathcal{R}_{K, K^{\prime}, 2}^{-\left(\rho-\rho_{0}\right)}[r] \otimes \mathcal{M}_{2}(\mathbb{C})$;

- the map $\mathfrak{C}^{\theta}(U)$ satisfies, for any $0 \leq k \leq K-K^{\prime}, V \in C_{* \mathbb{R}}^{K-K^{\prime}}\left(I ; \dot{H}^{s}\left(\mathbb{T} ; \mathbb{C}^{2}\right)\right)$,

$$
\begin{aligned}
& \left\|\partial_{t}^{k} \mathfrak{C}^{\theta}(U)[V]\right\|_{\dot{H}^{s-\frac{3}{2} k}}+\left\|\partial_{t}^{k}\left(\mathfrak{C}^{-\theta}(U)\right)^{-1}[V]\right\|_{\dot{H}^{s-\frac{3}{2} k}} \\
& \leq\|V\|_{k, s}\left(1+C_{s, r, K}\|U\|_{K, s_{0}}\right)+C_{s, r, K}\|V\|_{k, s_{0}}\|U\|_{K, s},
\end{aligned}
$$

uniformly in $\theta \in[0,1]$. Moreover the map $\mathfrak{C}^{\theta}(U)=U+\theta M_{1}(U)[U]+M_{\geq 2}(\theta ; U)[U]$ where $M_{1}(U)$ is in $\widetilde{\mathcal{M}}_{1} \otimes \mathcal{M}_{2}(\mathbb{C})$ and $M_{\geq 2}(\theta ; U) \in \mathcal{M}_{K, K^{\prime}, 2}[r] \otimes \mathcal{M}_{2}(\mathbb{C})$ with estimates uniform in $\theta \in[0,1]$.

In order to prove Proposition 4.3 we first provide lower bounds on the "small divisors" which appear in the Poincaré-Birkhoff reduction procedure.

\subsection{Three waves interactions}

We analyze the possible three waves interactions among the linear frequencies (1.5). We first notice that they admit an expansion as

$$
\Omega(n)=\sqrt{|n| \tanh (h|n|)\left(g+\kappa n^{2}\right)}=\sqrt{\kappa}|n|^{\frac{3}{2}}+r(n), \quad|r(n)| \leq C|n|^{-\frac{1}{2}}
$$

for some constant $C:=C(g, \kappa, h)>0$.

Lemma 4.4. (3-waves interactions) There exist $\mathrm{c}, \mathrm{C}>0$ such that for any $n_{1}, n_{2}, n_{3} \in \mathbb{Z} \backslash\{0\}$, $\sigma, \sigma^{\prime}= \pm$, such that

$$
n_{1}+\sigma n_{2}+\sigma^{\prime} n_{3}=0
$$

and $\max \left(\left|n_{1}\right|,\left|n_{2}\right|,\left|n_{3}\right|\right) \geq \mathrm{C}$, we have

$$
\left|\Omega\left(n_{1}\right)+\sigma \Omega\left(n_{2}\right)+\sigma^{\prime} \Omega\left(n_{3}\right)\right| \geq \mathrm{c} .
$$

If $\max \left(\left|n_{1}\right|,\left|n_{2}\right|,\left|n_{3}\right|\right)<\mathrm{C}$, then, either the phase $\Omega\left(n_{1}\right)+\sigma \Omega\left(n_{2}\right)+\sigma^{\prime} \Omega\left(n_{3}\right)$ is zero, or (4.14) holds. 
Proof. If $\sigma=\sigma^{\prime}=+$ then the bound (4.14 is trivial for all $n_{1}, n_{2}, n_{3} \in \mathbb{Z} \backslash\{0\}$. Assume $\sigma=-$ and $\sigma^{\prime}=-$ (the cases $\left(\sigma, \sigma^{\prime}\right)=(+,-)$ and $\left(\sigma, \sigma^{\prime}\right)=(-,+)$ are the same, up to reordering the indexes). Then, by (4.13), we have $n_{1}=n_{2}+n_{3}$ and we may suppose that $\left|n_{1}\right| \geq\left|n_{2}\right|,\left|n_{3}\right|$, otherwise the bound (4.14) is trivial. Without loss of generality we assume $n_{1}>0$, thus, also $n_{2}$ and $n_{3}$ are positive. In conclusion we assume that $n_{1} \geq n_{2} \geq n_{3} \geq 1$. By (4.12),

$$
\begin{aligned}
\left|\Omega\left(n_{1}\right)-\Omega\left(n_{2}\right)-\Omega\left(n_{3}\right)\right| & =\left|\Omega\left(n_{2}+n_{3}\right)-\Omega\left(n_{2}\right)-\Omega\left(n_{3}\right)\right| \\
& \geq \sqrt{\kappa}\left(\left(n_{2}+n_{3}\right)^{\frac{3}{2}}-n_{2}^{\frac{3}{2}}-n_{3}^{\frac{3}{2}}\right)-\frac{3 C}{\sqrt{n_{3}}} .
\end{aligned}
$$

Now

$$
\begin{aligned}
\left(n_{2}+n_{3}\right)^{\frac{3}{2}}-n_{2}^{\frac{3}{2}}-n_{3}^{\frac{3}{2}} & =\frac{\left(n_{2}+n_{3}\right)^{3}-\left(n_{2}^{\frac{3}{2}}+n_{3}^{\frac{3}{2}}\right)^{2}}{\left(n_{2}+n_{3}\right)^{\frac{3}{2}}+n_{2}^{\frac{3}{2}}+n_{3}^{\frac{3}{2}}}=\frac{3\left(n_{2}^{2} n_{3}+n_{2} n_{3}^{2}\right)-2 n_{2}^{\frac{3}{2}} n_{3}^{\frac{3}{2}}}{\left(n_{2}+n_{3}\right)^{\frac{3}{2}}+n_{2}^{\frac{3}{2}}+n_{3}^{\frac{3}{2}}} \\
& =\frac{9\left(n_{2}^{2} n_{3}+n_{2} n_{3}^{2}\right)^{2}-4 n_{2}^{3} n_{3}^{3}}{\left(n_{2}+n_{3}\right)^{\frac{3}{2}}+n_{2}^{\frac{3}{2}}+n_{3}^{\frac{3}{2}}} \frac{1}{3\left(n_{2}^{2} n_{3}+n_{2} n_{3}^{2}\right)+2 n_{2}^{\frac{3}{2}} n_{3}^{\frac{3}{2}}} \\
& =\frac{9\left(n_{2}^{4} n_{3}^{2}+n_{2}^{2} n_{3}^{4}\right)+14 n_{2}^{3} n_{3}^{3}}{\left(\left(n_{2}+n_{3}\right)^{\frac{3}{2}}+n_{2}^{\frac{3}{2}}+n_{3}^{\frac{3}{2}}\right)\left(3\left(n_{2}^{2} n_{3}+n_{2} n_{3}^{2}\right)+2 n_{2}^{\frac{3}{2}} n_{3}^{\frac{3}{2}}\right)} \\
& \geq \frac{9}{16(1+\sqrt{2})} \sqrt{n_{2}} \geq \frac{\sqrt{n_{2}}}{5}
\end{aligned}
$$

using that $n_{2} \geq n_{3} \geq 1$. By (4.15) and (4.16) we deduce that the phase

$$
\left|\Omega\left(n_{1}\right)-\Omega\left(n_{2}\right)-\Omega\left(n_{3}\right)\right| \geq \sqrt{n_{2}}\left(\frac{\sqrt{\kappa}}{5}-\frac{3 C}{\sqrt{n_{2} n_{3}}}\right) \geq \sqrt{n_{2}} \frac{\sqrt{\kappa}}{10}
$$

if $n_{2} n_{3} \geq(30 C)^{2} / \kappa$, in particular, since $n_{3} \geq 1$, if

$$
n_{2} \geq C_{1}:=(30 C)^{2} / \kappa \text {. }
$$

Recall that $n_{1}=n_{2}+n_{3} \leq 2 n_{2}$. Therefore $n_{2} \geq n_{1} / 2$ and we conclude that

$$
n_{1}=\max \left(n_{1}, n_{2}, n_{3}\right) \geq 2 C_{1} \Longrightarrow n_{2} \geq C_{1} \Longrightarrow\left|\Omega\left(n_{1}\right)-\Omega\left(n_{2}\right)-\Omega\left(n_{3}\right)\right| \geq \sqrt{n_{2}} \frac{\sqrt{\kappa}}{10}
$$

For the finitely many integers $n_{1}, n_{2}, n_{3}$ satisfying $\max \left(\left|n_{1}\right|,\left|n_{2}\right|,\left|n_{3}\right|\right) \leq \mathrm{C}:=2 C_{1}$ such that the phase $\Omega\left(n_{1}\right)-\Omega\left(n_{2}\right)-\Omega\left(n_{3}\right) \neq 0$, the lower bound (4.14) is trivial.

Remark 4.5. The constant $C(g, \kappa, h)$ in (4.12) is bounded by $c\left(\sqrt{\kappa} h^{-2}+g \kappa^{-1 / 2}\right)$, for some constant $c>0$ independent of $\kappa, g, h$. Then, there are $h_{0}, \kappa_{0}$ such that, if $h \geq h_{0}, \kappa>$ $\kappa_{0} g$, then (4.17) holds, for all $n_{1}, n_{2}, n_{3} \in \mathbb{Z} \backslash\{0\}$. As a consequence there are no 3-waves interactions, i.e. (4.14) holds for all $n_{1}, n_{2}, n_{3} \in \mathbb{Z} \backslash\{0\}$.

Notice that, for some values of the parameters $(g, \kappa, h)$, there could be 3 -waves interactions. 


\subsection{Poincaré-Birkhoff normal form of the smoothing quadratic terms}

In order to prove Proposition 4.3, we conjugate 4.1) with the flow

$$
\partial_{\theta} \mathfrak{C}^{\theta}(U)=\mathrm{G}_{1}(U) \mathfrak{C}^{\theta}(U), \quad \mathfrak{C}^{0}(U)=\mathrm{Id},
$$

with an operator $\mathrm{G}_{1}(U)$ in $\widetilde{\mathcal{R}}_{1}^{-\rho} \otimes \mathcal{M}_{2}(\mathbb{C})$, of the same form of $\mathrm{R}_{1}(U)$ in (4.4)-(4.7), to be determined. We introduce the new variable $Y:=\left[\frac{y}{y}\right]=\left(\mathfrak{C}^{\theta}(U)[Z]\right)_{\left.\right|_{\theta=1}}$.

Lemma 4.6. If $\mathrm{G}_{1}(U) \in \widetilde{\mathcal{R}}_{1}^{-\rho} \otimes \mathcal{M}_{2}(\mathbb{C})$ solves the homological equation

$$
\mathrm{G}_{1}(\mathrm{i} \Omega(D) E U)+\left[\mathrm{G}_{1}(U), \mathrm{i} \Omega(D) E\right]+\mathrm{R}_{1}(U)=\mathrm{R}_{1}^{\text {res }}(U),
$$

where $\mathrm{R}_{1}^{\text {res }}(U)$ is the Poincaré-Birkhoff resonant operator in Definition 4.2 then

$$
\partial_{t} Y=\mathrm{i} \Omega(D) E Y+\mathrm{R}_{1}^{r e s}(U)[Y]+\mathrm{i} O p^{\mathrm{BW}}\left(\mathcal{H}_{\geq 2}(U ; \xi)\right) Y+\mathrm{R}_{\geq 2}(U)[Y]
$$

where $\mathcal{H}_{\geq 2}(U ; \xi)$ is the same diagonal matrix of symbols in (4.2) and $\mathrm{R}_{\geq 2}(U)$ is a real-to-real smoothing operator in $\mathcal{R}_{K, K^{\prime}, 2}^{-\rho+m_{1}}[r] \otimes \mathcal{M}_{2}(\mathbb{C})$ with $m_{1} \geq 3 / 2$ (fixed below (3.8)).

The flow map $\mathfrak{C}^{\theta}(U)$ in (4.18) satisfies (4.11) and $\mathfrak{C}^{\theta}(U)=U+\theta M_{1}(U)[U]+M_{\geq 2}(\theta ; U)[U]$ where $M_{1}(U)$ is in $\widetilde{\mathcal{M}}_{1} \otimes \mathcal{M}_{2}(\mathbb{C})$ and $M_{\geq 2}(\theta ; U) \in \mathcal{M}_{K, K^{\prime}, 2}[r] \otimes \mathcal{M}_{2}(\mathbb{C})$ with estimates uniform in $\theta \in[0,1]$.

Proof. Since $\mathrm{G}_{1}(U)$ is a smoothing operator then the flow in 4.18 is well-posed in Sobolev spaces and satisfies the estimates (4.11), as well as the last statement, by e.g. Lemma A.3 in [7]. To conjugate (4.1) we apply the usual Lie expansion up to the first order (see for instance Lemma $A .1$ in [7]). Denoting $\operatorname{Ad}_{\mathrm{G}_{1}}:=\left[\mathrm{G}_{1}\right.$, ], we have

$$
\begin{aligned}
\mathfrak{C}^{1}(U) \Omega(D) E\left(\mathfrak{C}^{1}(U)\right)^{-1} & =\Omega(D) E+\left[\mathrm{G}_{1}(U), \Omega(D) E\right] \\
& +\int_{0}^{1}(1-\theta) \mathfrak{C}^{\boldsymbol{\theta}}(U) \operatorname{Ad}_{\mathrm{G}_{1}(U)}^{2}[\Omega(D) E]\left(\mathfrak{C}^{-\theta}(U)\right)^{-1} d \theta .
\end{aligned}
$$

Using that $\mathrm{G}_{1}(U)$ belongs to $\widetilde{\mathcal{R}}_{1}^{-\rho} \otimes \mathcal{M}_{2}(\mathbb{C})$, Proposition 2.7 and 4.11), the integral term in (4.21) is a smoothing operator in $\mathcal{R}_{K, K^{\prime}, 2}^{-\rho+\frac{3}{2}}[r] \otimes \mathcal{M}_{2}(\mathbb{C})$. Similarly, we obtain

$$
\mathfrak{C}^{1}(U) O p^{\mathrm{BW}}\left(\mathcal{H}_{\geq 2}(U ; \xi)\right)\left(\mathfrak{C}^{1}(U)\right)^{-1}=O p^{\mathrm{BW}}\left(\mathcal{H}_{\geq 2}(U ; \xi)\right)
$$

up to a matrix of smoothing operators in $\mathcal{R}_{K, K^{\prime}, 2}^{-\rho+\frac{3}{2}}[r] \otimes \mathcal{M}_{2}(\mathbb{C})$. Finally

$$
\mathfrak{C}^{1}(U)\left(\mathrm{R}_{1}(U)+\mathrm{R}_{\geq 2}(U)\right)\left(\mathfrak{C}^{1}(U)\right)^{-1}=\mathrm{R}_{1}(U)
$$

plus a smoothing operator in $\mathcal{R}_{K, K^{\prime}, 2}^{-\rho}[r] \otimes \mathcal{M}_{2}(\mathbb{C})$.

Next we consider the contribution coming from the conjugation of $\partial_{t}$. Applying again a Lie expansion formula (see Lemma $A .1$ in [7]) we get

$$
\begin{aligned}
& \partial_{t} \mathfrak{C}^{1}(U)\left(\mathfrak{C}^{1}(U)\right)^{-1}=\partial_{t} \mathrm{G}_{1}(U)+ \\
& \frac{1}{2}\left[\mathrm{G}_{1}(U), \partial_{t} \mathrm{G}_{1}(U)\right]+\frac{1}{2} \int_{0}^{1}(1-\theta)^{2} \mathfrak{C}^{-\theta}(U) \operatorname{Ad}_{\mathfrak{G}_{1}(U)}^{2}\left[\partial_{t} \mathrm{G}_{1}(U)\right]\left(\mathfrak{C}^{\theta}(U)\right)^{-1} d \theta
\end{aligned}
$$


Recalling (3.8) we have

$$
\partial_{t} \mathrm{G}_{1}(U)=\mathrm{G}_{1}(\mathrm{i} \Omega(D) E U+\mathrm{i} M(U)[U])=\mathrm{G}_{1}(\mathrm{i} \Omega(D) E U)
$$

up to a term in $\mathcal{R}_{K, K^{\prime}, 2}^{-\rho+m_{1}}[r] \otimes \mathcal{M}_{2}(\mathbb{C})$, where we used Proposition 2.7. By [4.23), the fact that $\mathrm{G}_{1}(\mathrm{i} \Omega(D) E U)$ is in $\widetilde{\mathcal{R}}_{1}^{-\rho+(3 / 2)} \otimes \mathcal{M}_{2}(\mathbb{C})$ and (4.11), we deduce that the term in 4.22) belongs to $\Sigma \mathcal{R}_{K, K^{\prime}, 2}^{-\rho+m_{1}}[r, N] \otimes \mathcal{M}_{2}(\mathbb{C})$. Collecting all the previous expansions, and using that $\mathrm{G}_{1}(U)$ solves 4.19), we deduce 4.20.

We now solve the homological equation 4.19.

Lemma 4.7. (Homological equation) Let $\mathrm{G}_{1}(U)$ be an operator of the form (4.4)-(4.7) with coefficients

$$
\left(\mathrm{g}_{1, \epsilon}\right)_{n, k}^{\sigma, \sigma^{\prime}}:=\frac{\left(\mathrm{r}_{1, \epsilon}\right)_{n, k}^{\sigma, \sigma^{\prime}}}{\mathrm{i}\left(\sigma \Omega(j)-\sigma^{\prime} \Omega(k)-\epsilon \Omega(n)\right)},
$$

for any $\sigma, \sigma^{\prime}, \epsilon= \pm, j, n, k \in \mathbb{Z} \backslash\{0\}$, satisfying

$$
\sigma j-\sigma^{\prime} k-\epsilon n=0, \quad \sigma \Omega(j)-\sigma^{\prime} \Omega(k)-\epsilon \Omega(n) \neq 0,
$$

and $\left(\mathrm{g}_{1, \epsilon}\right)_{n, k}^{\sigma, \sigma^{\prime}}:=0$ otherwise. Then $\mathrm{G}_{1}(U)$ is in $\widetilde{\mathcal{R}}_{1}^{-\rho} \otimes \mathcal{M}_{2}(\mathbb{C})$ and solves the homological equation 4.19).

Proof. The coefficients in (4.24) are well defined by (4.25) and, by Lemma 4.4, they satisfy the uniform lower bound $\left|\sigma \Omega(j)-\sigma^{\prime} \Omega(k)-\epsilon \Omega(n)\right| \geq \mathrm{c}$. Then the operator $\mathrm{G}_{1}(U)$ is in $\widetilde{\mathcal{R}}_{1}^{-\rho} \otimes \mathcal{M}_{2}(\mathbb{C})$, see e.g. Lemma 6.5 of [7].

Next, recalling (4.4), the homological equation (4.19) amounts to the equations

$$
\left(\mathrm{G}_{1}(\mathrm{i} \Omega(D) E U)\right)_{\sigma}^{\sigma^{\prime}}+\left(\mathrm{G}_{1}(U)\right)_{\sigma}^{\sigma^{\prime}} \sigma^{\prime} \mathrm{i} \Omega(D)-\sigma \mathrm{i} \Omega(D)\left(\mathrm{G}_{1}(U)\right)_{\sigma}^{\sigma^{\prime}}+\left(\mathrm{R}_{1}(U)\right)_{\sigma}^{\sigma^{\prime}}=\left(\mathrm{R}_{1}^{\text {res }}(U)\right)_{\sigma}^{\sigma^{\prime}}
$$

for $\sigma, \sigma^{\prime}= \pm$, and, setting $\mathrm{F}_{1}(U):=\mathrm{G}_{1}(\mathrm{i} \Omega(D) E U)$ to the equations, for any $j, k \in \mathbb{Z} \backslash\{0\}$, $\epsilon= \pm$

$$
\begin{aligned}
& \left(\mathrm{F}_{1, \epsilon}(U)\right)_{\sigma, j}^{\sigma^{\prime}, k}+\left(\mathrm{G}_{1, \epsilon}(U)\right)_{\sigma, j}^{\sigma^{\prime}, k}\left(-\sigma \mathrm{i} \Omega(j)+\sigma^{\prime} \mathrm{i} \Omega(k)\right)+\left(\mathrm{R}_{1, \epsilon}(U)\right)_{\sigma, j}^{\sigma^{\prime}, k} \\
& =\left(\mathrm{R}_{1, \epsilon}^{r e s}(U)\right)_{\sigma, j}^{\sigma^{\prime}, k} .
\end{aligned}
$$

Expanding $\left(\mathrm{G}_{1}(U)\right)_{\sigma}^{\sigma^{\prime}}$ as in (4.5)-(4.7) with entries

$$
\left(\mathrm{G}_{1, \epsilon}(U)\right)_{\sigma, j}^{\sigma^{\prime}, k}=\frac{1}{\sqrt{2 \pi}} \sum_{\substack{n \in \mathbb{Z} \backslash\{0\} \\ \epsilon n+\sigma^{\prime} k=\sigma j}}\left(\mathrm{~g}_{1, \epsilon}\right)_{n, k}^{\sigma, \sigma^{\prime}} u_{n}^{\epsilon}, \quad j, k \in \mathbb{Z} \backslash\{0\},
$$

we have that $\mathrm{F}_{1}(U):=\mathrm{G}_{1}(\mathrm{i} \Omega(D) E U)$ satisfies

$$
\left(\mathrm{F}_{1, \epsilon}(U)\right)_{\sigma, j}^{\sigma^{\prime}, k}=\frac{1}{\sqrt{2 \pi}} \sum_{n \in \mathbb{Z} \backslash\{0\}, \epsilon n+\sigma^{\prime} k=\sigma j}\left(\mathrm{~g}_{1, \epsilon}\right)_{n, k}^{\sigma, \sigma^{\prime}}(\mathrm{i} \Omega(n) \epsilon) u_{n}^{\epsilon} .
$$


Hence the left hand side in (4.26) has coefficients

$$
-\left(\mathrm{g}_{1, \epsilon}\right)_{n, k}^{\sigma, \sigma^{\prime}} \mathrm{i}\left(\sigma \Omega(j)-\sigma^{\prime} \Omega(k)-\epsilon \Omega(n)\right)+\left(\mathrm{r}_{1, \epsilon}\right)_{n, k}^{\sigma, \sigma^{\prime}}
$$

for $j, k, n \in \mathbb{Z} \backslash\{0\}$ and $\sigma, \sigma^{\prime}, \epsilon= \pm$ with $\epsilon n+\sigma^{\prime} k=\sigma j$. Recalling Definition 4.2 we deduce that $\mathrm{G}_{1}(U)$ with coefficients in (4.24) solves the homological equation 4.19).

Proof of Proposition 4.3. We apply Lemmata 4.6 and 4.7. The change of variables that transforms (4.1) into (4.20) is $Y=\mathfrak{C}^{\theta}(U) Z$ where $\mathfrak{C}^{\theta}(U)$ is the flow map in 4.18) that satisfies (4.11) and the last statement in Lemma 4.6. Moreover, using also the last item of Proposition 3.2 we may express

$$
\begin{aligned}
& Y=\left(\mathfrak{C}^{\theta}(U) \circ \mathfrak{F}^{\theta}(U)\right)_{\left.\right|_{\theta=1}}[U]=U+\widetilde{\mathrm{M}}(U)[U], \\
& \widetilde{\mathrm{M}}(U) \in \Sigma \mathcal{M}_{K, K^{\prime}, 1}^{m_{2}}[r, 2] \otimes \mathcal{M}_{2}(\mathbb{C}), m_{2} \geq 3 / 2 .
\end{aligned}
$$

Then system (4.20) can be written as system 4.9) with $\mathcal{X}_{\geq 3}(U, Y)$ given in (4.10) and

$$
\mathfrak{R}_{\geq 2}(U):=\mathrm{R}_{1}^{\text {res }}(U)-\mathrm{R}_{1}^{\text {res }}(U+\widetilde{\mathrm{M}}(U)[U])+\mathrm{R}_{\geq 2}(U) .
$$

By (4.27) and Proposition 2.7-(iii) we have that $\mathfrak{R}_{\geq 2}(U) \in \Sigma \mathcal{R}_{K, K^{\prime}, 2}^{-\left(\rho-\rho_{0}\right)} \otimes \mathcal{M}_{2}(\mathbb{C})$ where $\rho_{0}:=\max \left\{m_{1}, m_{2}\right\}$.

\section{Birkhoff normal form and quadratic life-span of solutions}

In this section we prove Theorems 1.1 and 1.2. We first recall the Hamiltonian formalism in the complex symplectic variables

$$
\left(\begin{array}{l}
u \\
\bar{u}
\end{array}\right):=\mathrm{B}\left(\begin{array}{l}
\eta \\
\psi
\end{array}\right)=\frac{1}{\sqrt{2}}\left(\begin{array}{c}
\Lambda \psi+\mathrm{i} \Lambda^{-1} \eta \\
\Lambda \psi-\mathrm{i} \Lambda^{-1} \eta
\end{array}\right),\left(\begin{array}{l}
\eta \\
\psi
\end{array}\right):=\mathrm{B}^{-1}\left(\begin{array}{c}
u \\
\bar{u}
\end{array}\right)=\frac{1}{\sqrt{2}}\left(\begin{array}{c}
-\mathrm{i} \Lambda(u-\bar{u}) \\
\Lambda^{-1}(u+\bar{u})
\end{array}\right),
$$

where $\Lambda$ is the Fourier multiplier defined in 3.2 .

A vector field $X(\eta, \psi)$ and a function $H(\eta, \psi)$ assume the form

$$
X^{\mathbb{C}}:=\mathrm{B}^{\star} X:=\mathrm{B} X \mathrm{~B}^{-1}, \quad H_{\mathbb{C}}:=H \circ \mathrm{B}^{-1} .
$$

The Poisson bracket in (1.3) reads $\left\{F_{\mathbb{C}}, H_{\mathbb{C}}\right\}:=\mathrm{i} \sum_{j \in \mathbb{Z} \backslash\{0\}} \partial_{u_{j}} H_{\mathbb{C}} \partial_{\overline{u_{j}}} F_{\mathbb{C}}-\partial_{\overline{u_{j}}} H_{\mathbb{C}} \partial_{u_{j}} F_{\mathbb{C}}$.

Given a Hamiltonian $F_{\mathbb{C}}$, expressed in the complex variables $(u, \bar{u})$, the associated Hamiltonian vector field $X_{F_{\mathbb{C}}}$ is

$$
X_{F_{\mathbb{C}}}=\left(\begin{array}{c}
\mathrm{i} \partial_{\bar{u}} F_{\mathbb{C}} \\
-\mathrm{i} \partial_{u} F_{\mathbb{C}}
\end{array}\right)=\frac{1}{\sqrt{2 \pi}} \sum_{k \in \mathbb{Z} \backslash\{0\}}\left(\begin{array}{c}
\mathrm{i} \partial_{\overline{u_{k}}} F_{\mathbb{C}} e^{\mathrm{i} k x} \\
-\mathrm{i} \partial_{u_{k}} F_{\mathbb{C}} e^{-\mathrm{i} k x}
\end{array}\right)
$$

that we also identify, using the standard vector field notation, with

$$
X_{F_{\mathbb{C}}}=\sum_{k \in \mathbb{Z} \backslash\{0\}, \sigma= \pm} \mathrm{i} \sigma \partial_{u_{k}^{-\sigma}} F_{\mathbb{C}} \partial_{u_{k}^{\sigma}}
$$


If $X_{F}$ is the Hamiltonian vector field of the Hamiltonian $F:=F_{\mathbb{C}} \circ \mathrm{B}$, we have

$$
X_{F}^{\mathbb{C}}:=\mathrm{B}^{\star} X_{F}=X_{F_{\mathbb{C}}} .
$$

The push-forward acts naturally on the commutator of nonlinear vector fields, defined in (5.14), namely

$$
B^{\star} \llbracket X, Y \rrbracket=\llbracket B^{\star} X, B^{\star} Y \rrbracket=\llbracket X^{\mathbb{C}}, Y^{\mathbb{C}} \rrbracket .
$$

Recalling (1.6), the Hamiltonian (1.2) admits, in complex coordinates, the expansion

$$
H_{\mathbb{C}}:=H \circ \mathrm{B}^{-1}=H_{\mathbb{C}}^{(2)}+H_{\mathbb{C}}^{(3)}+\ldots
$$

where, recalling (3.3), 1.5), (2.2),

$$
H_{\mathbb{C}}^{(2)}=\sum_{j \in \mathbb{Z} \backslash\{0\}} \Omega(j) u_{j} \overline{u_{j}}, \quad H_{\mathbb{C}}^{(3)}=\sum_{\sigma_{1} j_{1}+\sigma_{2} j_{2}+\sigma_{3} j_{3}=0} H_{j_{1}, j_{2}, j_{3}}^{\sigma_{1}, \sigma_{2}} u_{j_{1}}^{\sigma_{1}} u_{j_{2}}^{\sigma_{2}} u_{j_{3}}^{\sigma_{3}}
$$

and $H_{j_{1}, j_{2}, j_{3}}^{\sigma_{1}, \sigma_{2}, \sigma_{3}}$ are computed in (1.12), for $j_{1}, j_{2}, j_{3} \in \mathbb{Z} \backslash\{0\}$.

\subsection{Normal form identification and proof of Theorem 1.1}

A normal form uniqueness argument allows to identify the quadratic Poincaré-Birkhoff resonant vector field $\mathrm{R}_{1}^{\text {res }}(Y)[Y]$ in 4.9 as the cubic resonant Hamiltonian vector field obtained by the formal Birkhoff normal form construction in [14].

Proposition 5.1. (Identification of the quadratic resonant Birkhoff normal form) The Birkhoff resonant vector field $\mathrm{R}_{1}^{\text {res }}(Y)[Y]$ defined in (4.9) is equal to

$$
\mathrm{R}_{1}^{\text {res }}(Y)[Y]=X_{H_{B N F}^{(3)}}
$$

where $H_{B N F}^{(3)}$ is the cubic Birkhoff normal form Hamiltonian in (1.11).

The proof follows the ideas developed in Section 7 in [7]. Recalling [1.6], we first expand the water waves Hamiltonian vector field in 1.1 -1.2 in degrees of homogeneity

$$
X_{H}=X_{1}+X_{2}+X_{\geq 3} \quad \text { where } \quad X_{1}:=X_{H^{(2)}}, X_{2}:=X_{H^{(3)}},
$$

and $X_{\geq 3}$ collects the higher order terms. System 4.9) has been obtained conjugating (1.1) under the map

$$
Y=\mathbf{F}^{1}(U) \circ \mathrm{B} \circ \mathcal{G}\left[\begin{array}{l}
\eta \\
\psi
\end{array}\right],
$$

where $\mathcal{G}$ is the good-unknown transformation (see (3.1)

$$
\left[\begin{array}{l}
\eta \\
\omega
\end{array}\right]=\mathcal{G}\left[\begin{array}{l}
\eta \\
\psi
\end{array}\right]:=\left[\begin{array}{c}
\eta \\
\psi-O p^{\mathrm{BW}^{2}}(B(\eta, \psi)) \eta
\end{array}\right],
$$

the map B is defined in (5.1) (see (3.3) and Proposition 3.1), and

$$
\mathbf{F}^{\theta}(U):=\mathfrak{C}^{\theta}(U) \circ \mathfrak{F}^{\theta}(U), \quad \theta \in[0,1],
$$


where $\mathfrak{F}^{\theta}(U), \mathfrak{C}^{\theta}(U)$ are defined respectively in Propositions 3.2 and 4.3 In order to identify the quadratic vector field in system (4.9), we perform a Lie commutator expansion, up to terms of homogeneity at least 3 . Notice that the quadratic term in 4.9) may arise by only the conjugation of $X_{1}+X_{2}$ under the homogeneous components of the paradifferential transformations $\mathcal{G}$ and $\mathbf{F}^{1}(U)$, neglecting cubic terms.

We use the following Lemma 5.2 that collects Lemmata A.8, A.9 and A.10 in [7]. The variable $U$ may denote both the couple of complex variables $(u, \bar{u})$ or the real variables $(\eta, \psi)$.

Lemma 5.2 ( [7]). (Lie expansion) Consider a map $\theta \mapsto \mathbf{F}_{\leq 2}^{\theta}(U), \theta \in[0,1]$, of the form

$$
\mathbf{F}_{\leq 2}^{\theta}(U)=U+\theta M_{1}(U)[U], \quad M_{1}(U) \in \widetilde{\mathcal{M}}_{1} \otimes \mathcal{M}_{2}(\mathbb{C}) .
$$

Then:

(i) the family of maps $\mathbf{G}_{\leq 2}^{\theta}(V):=V-\theta M_{1}(V)[V]$ is such that

$$
\mathbf{G}_{\leq 2}^{\theta} \circ \mathbf{F}_{\leq 2}^{\theta}(U)=U+M_{\geq 2}(\theta ; U)[U], \quad \mathbf{F}_{\leq 2}^{\theta} \circ \mathbf{G}_{\leq 2}^{\theta}(V)=V+M_{\geq 2}(\theta ; U)[U],
$$

where $M_{\geq 2}(\theta ; U)$ is a polynomial in $\theta$ and finitely many monomials $M_{p}(U)[U]$ for $M_{p}(U) \in$ $\widetilde{\mathcal{M}}_{p} \otimes \mathcal{M}_{2}(\mathbb{C}), p \geq 2$;

(ii) the family of maps $\mathbf{G}_{\leq 2}^{\theta}(V)$ satisfies

$$
\partial_{\theta} \mathbf{G}_{\leq 2}^{\theta}(V)=S\left(\mathbf{G}_{\leq 2}^{\theta}(V)\right)+M_{\geq 2}(\theta ; U)[U], \quad \mathbf{G}_{\leq 2}^{0}(V)=V,
$$

where $S(U)=S_{1}(U)[U]$ with $S_{1}(U) \in \widetilde{\mathcal{M}}_{1} \otimes \mathcal{M}_{2}(\mathbb{C})$ and $M_{\geq 2}(\theta ; U)$ is a polynomial in $\theta$ and finitely many monomials $M_{p}(U)[U]$ for maps $M_{p}(U) \in \widetilde{\mathcal{M}}_{p} \otimes \mathcal{M}_{2}(\mathbb{C}), p \geq 2$.

(iii) Let $X(U)=M(U) U$ for some map $M(U)=M_{0}+M_{1}(U)$ where $M_{0}$ is in $\widetilde{\mathcal{M}}_{0} \otimes \mathcal{M}_{2}(\mathbb{C})$ and $M_{1}(U)$ in $\widetilde{\mathcal{M}}_{1} \otimes \mathcal{M}_{2}(\mathbb{C})$. If $U$ solves $\partial_{t} U=X(U)$, then the function $V:=\mathbf{F}_{\leq 2}^{1}(U)$ solves

$$
\partial_{t} V=X(V)+\llbracket S, X \rrbracket(V)+\cdots,
$$

up to terms of degree of homogeneity greater or equal to 3 , where we define the nonlinear commutator

$$
\llbracket S, X \rrbracket(U):=d_{U} X(U)[S(U)]-d_{U} S(U)[X(U)] .
$$

- Notation. Given a homogeneous vector field $X$, we denote by $\Phi_{S}^{\star} X$ the induced (formal) push forward (see (5.13)

$$
\Phi_{S}^{\star} X=X+\llbracket S, X \rrbracket+\cdots
$$

where the dots $\cdots$ denote cubic terms.

\section{Proof of Proposition 5.1.}

Step 1. The good unknown change of variable $\mathcal{G}$ in $(5.10)$. First of all we note that $\mathcal{G}(\eta, \psi)=$ $\left(\Phi^{\theta}(\eta, \psi)\right)_{\theta=1}$ where

$$
\Phi^{\theta}\left[\begin{array}{l}
\eta \\
\psi
\end{array}\right]=\left[\begin{array}{c}
\stackrel{\eta}{\psi} \\
\psi-\theta O p^{\mathrm{BW}} \\
(B(\eta, \psi)) \eta
\end{array}\right], \quad \theta \in[0,1] .
$$


Since $B(\eta, \psi)$ is a function in $\Sigma \mathcal{F}_{K, 0,1}^{\mathbb{R}}[r, 2]$ we have that $\Phi^{\theta}(\eta, \psi)$ has an expansion as in (5.12) up to cubic terms. Hence, by Lemma 5.2 $-(i)-(i i)$, we regard the inverse of the map $\mathcal{G}_{\leq 2}$, obtained truncating $\mathcal{G}$ up to cubic remainders, as the (formal) time one flow of a quadratic vector field

$$
\mathrm{S}_{2}:=S_{1}(\eta, \psi)\left[\begin{array}{l}
\eta \\
\psi
\end{array}\right], \quad S_{1}(\eta, \psi) \in \widetilde{\mathcal{M}}_{1} \otimes \mathcal{M}_{2}(\mathbb{C}) .
$$

By (5.8), (5.15) and (5.16), we get

$$
\Phi_{\mathrm{S}_{2}}^{\star}\left(X_{1}+X_{2}\right)=X_{1}+X_{2}+\llbracket \mathrm{S}_{2}, X_{1} \rrbracket+\cdots .
$$

Step 2. Complex coordinates B in (5.1). In the complex coordinates (5.1), the vector field (5.17) reads, recalling (5.2) and (5.5),

$$
\mathrm{B}^{\star} \Phi_{\mathrm{S}_{2}}^{\star}\left(X_{1}+X_{2}\right)=X_{1}^{\mathbb{C}}+X_{2}^{\mathbb{C}}+\llbracket \mathrm{S}_{2}^{\mathbb{C}}, X_{1}^{\mathbb{C}} \rrbracket+\cdots
$$

where, by (5.4), (5.8), (5.6),

$$
X_{1}^{\mathbb{C}}=X_{H_{\mathbb{C}}^{(2)}}=\mathrm{i} \sum_{j, \sigma} \sigma \Omega(j) u_{j}^{\sigma} \partial_{u_{j}^{\sigma}}, \quad X_{2}^{\mathbb{C}}=X_{H_{\mathbb{C}}^{(3)}} .
$$

Step 3. The transformation $\mathbf{F}^{1}$ in (5.11). By the last items of Proposition 3.2 and Proposition 4.3, the map $\mathbf{F}^{\theta}(U)$ has the form (5.12) up to cubic terms. Thus, by Lemma 5.2- $(i)-(i i)$, the approximate inverse of the truncated map $\mathbf{F}_{\leq 2}^{1}$ can be regarded as the (formal) time-one flow of a vector field

$$
\mathrm{T}_{2}:=T_{1}(U)[U], \quad T_{1}(U) \in \widetilde{\mathcal{M}}_{1} \otimes \mathcal{M}_{2}(\mathbb{C}) .
$$

By 5.18, 5.19), 5.15, we get

$$
\Phi_{\mathrm{T}_{2}}^{\star} \mathrm{B}^{\star} \Phi_{\mathrm{S}_{2}}^{\star}\left(X_{1}+X_{2}\right)=X_{H_{\mathbb{C}}^{(2)}}+X_{H_{\mathbb{C}}^{(3)}}+\llbracket \mathrm{S}_{2}^{\mathbb{C}}+\mathrm{T}_{2}, X_{H_{\mathbb{C}}^{(2)}} \rrbracket+\cdots .
$$

Comparing (4.9) and (5.21) we deduce that

$$
\mathrm{R}_{1}^{\text {res }}(Y)[Y] \equiv X_{H_{\mathbb{C}}^{(3)}}+\llbracket \mathrm{S}_{2}^{\mathbb{C}}+\mathrm{T}_{2}, X_{H_{\mathbb{C}}^{(2)}} \rrbracket .
$$

The vector field $\mathrm{R}_{1}^{\text {res }}(Y)[Y]$ is in Poincaré-Birkhoff normal form, recall Definition 4.2. Therefore, defining the linear operator $\Pi_{\text {ker }}$ acting on a quadratic monomial vector field $u_{j_{1}}^{\sigma_{1}} u_{j_{2}}^{\sigma_{2}} \partial_{u_{j}^{\sigma}}$ as

$$
\Pi_{\text {ker }}\left(u_{j_{1}}^{\sigma_{1}} u_{j_{2}}^{\sigma_{2}} \partial_{u_{j}^{\sigma}}\right):= \begin{cases}u_{j_{1}}^{\sigma_{1}} u_{j_{2}}^{\sigma_{2}} \partial_{u_{j}^{\sigma}} & \text { if }-\sigma \Omega(j)+\sigma_{1} \Omega\left(j_{1}\right)+\sigma_{2} \Omega\left(j_{2}\right)=0 \\ 0 & \text { otherwise }\end{cases}
$$

we have that

$$
\mathrm{R}_{1}^{r e s}(Y)[Y]=\Pi_{\text {ker }}\left(\mathrm{R}_{1}^{r e s}(Y)[Y]\right)
$$

In addition, since

$$
\llbracket u_{j_{1}}^{\sigma_{1}} u_{j_{2}}^{\sigma_{2}} \partial_{u_{j}^{\sigma}}, X_{H_{\mathbb{C}}^{(2)}} \rrbracket=\mathrm{i}\left(\sigma \Omega(j)-\sigma_{1} \Omega\left(j_{1}\right)-\sigma_{2} \Omega\left(j_{2}\right)\right) u_{j_{1}}^{\sigma_{1}} u_{j_{2}}^{\sigma_{2}} \partial_{u_{j}^{\sigma}},
$$


we deduce

$$
\Pi_{\mathrm{ker}} \llbracket \mathrm{S}_{2}^{\mathbb{C}}+\mathrm{T}_{2}, X_{H_{\mathbb{C}}^{(2)}} \rrbracket=0 .
$$

In conclusion, (5.24), (5.22) and (5.25) imply that

$$
\mathrm{R}_{1}^{r e s}(Y)[Y]=\Pi_{\mathrm{ker}}\left(X_{H_{\mathbb{C}}^{(3)}}\right) \stackrel{\sqrt[5.6]{=}}{=} X_{H_{B N F}^{(3)}}
$$

where $H_{B N F}^{(3)}$ is the Hamiltonian in (1.11). This proves (5.7).

Proof of Theorem 1.1. Hypothesis (1.8) implies that the variable $u$ defined in (3.3) satisfies (3.5) and therefore the function $U=\left[\frac{u}{u}\right]$ belongs to the ball $B_{s}^{K}(I ; r)$ (recall (2.1)) with $r=C_{s, K} \bar{\varepsilon} \ll 1$ and $I=[-T, T]$. By Proposition 3.1 the function $U$ solves system (3.6). Then we apply Proposition 3.2 and the Poincaré-Birkhoff Proposition 4.3 with $s \gg K \geq K^{\prime}(\rho)$ and $K^{\prime}(\rho)$ given by Proposition 3.2, taking $\bar{\varepsilon}$ small enough. The map $\mathbf{F}^{1}(U)$ in (5.11) transforms the water waves system (3.6) into (4.9), which, thanks to Proposition 5.1, is expressed in terms of the Hamiltonian $H_{B N F}^{(3)}$ in 1.11 as

$$
\partial_{t} y=\mathrm{i} \Omega(D) y+\mathrm{i} \partial_{\bar{y}} H_{B N F}^{(3)}(y, \bar{y})+\mathcal{X}_{\geq 3}^{+}
$$

where $\mathcal{X}_{\geq 3}^{+}$is the first component of $\mathcal{X}_{\geq 3}(U, Y)$ in (4.10). Renaming $y \rightsquigarrow z$, the above equation is (1.10). We define $z=\mathfrak{B}(\eta, \psi)[\eta, \psi]$ as the first component of the change of variable [5.9), namely of $\mathbf{F}^{1}(U) \circ \mathrm{B} \circ \mathcal{G}[\eta, \psi]$, with $U$ written in terms of $(\eta, \psi)$ by (3.3), (3.1). By (4.11) and (3.10) with $k=0$, and using that $U \in B_{s}^{K}(I ; r)$, we get

$$
\|z(t)\|_{\dot{H}^{s}} \sim_{s}\|u(t)\|_{\dot{H}^{s}},
$$

and (1.9) follows, using also (3.3), (3.1). The cubic vector field $\mathcal{X}_{\geq 3}^{+}$in (4.10) satisfies the estimate $\left\|\mathcal{X}_{\geq 3}^{+}\right\|_{\dot{H}^{s-\frac{3}{2}}} \lesssim_{s}\|z\|_{\dot{H}^{s}}^{3}$ by Proposition 3.8 in [6] (recall that $\mathcal{H}_{\geq 2}(U ; \xi) \in \Gamma_{K, K^{\prime}, 2}^{3 / 2}[r] \otimes$ $\mathcal{M}_{2}(\mathbb{C})$ ), by (2.10) with $k=0$, and (3.4), (5.26). Moreover, the vector field $\mathcal{X}_{\geq 3}^{+}$satisfies the energy estimate (1.13) since the symbol $\mathcal{H}_{\geq 2}(U ; \xi)$ is independent of $x$ and purely imaginary up to symbols of order 0 , see (4.3) (for the detailed argument we refer to Lemma 7.5 in [7]).

\subsection{Energy estimate and proof of Theorem 1.2}

We now deduce Theorem 1.2 by Theorem 1.1 and the following energy estimate for the solution $z$ of the Birkhoff resonant system (1.10). By time reversibility, without loss of generality, we may only look at positive times $t>0$.

Lemma 5.3. (Energy estimate) Fix $s, \bar{\varepsilon}>0$ as in Theorem 1.1 and assume that the solution $(\eta, \psi)$ of (1.1) satifies (1.8). Then the solution $z(t)$ of (1.10) satisfies

$$
\|z(t)\|_{\dot{H}^{s}}^{2} \leq C(s)\|z(0)\|_{\dot{H}^{s}}^{2}+C(s) \int_{0}^{t}\|z(\tau)\|_{\dot{H}^{s}}^{4} d \tau, \quad \forall t \in[0, T] .
$$


Proof. By Lemma 4.4, the Birkhoff resonant Hamiltonian $H_{B N F}^{(3)}$ in (1.11) depends on finitely many variables $z_{j_{1}}^{ \pm}, z_{j_{2}}^{ \pm}, z_{j_{3}}^{ \pm}, j_{1}, j_{2}, j_{3} \in \mathbb{Z} \backslash\{0\}$, because

$$
\left\{\begin{array}{l}
\sigma_{1} j_{1}+\sigma_{2} j_{2}+\sigma_{3} j_{3}=0 \\
\sigma_{1} \Omega\left(j_{1}\right)+\sigma_{2} \Omega\left(j_{2}\right)+\sigma_{3} \Omega\left(j_{3}\right)=0
\end{array} \quad \Longrightarrow \quad \max \left(\left|j_{1}\right|,\left|j_{2}\right|,\left|j_{3}\right|\right)<\mathrm{C} .\right.
$$

For any function $w \in \dot{H}^{s}(\mathbb{T})$ we define the projector $\Pi_{L}$ on low modes, respectively the projector $\Pi_{H}$ on high modes, as

$$
w_{L}:=\Pi_{L} w:=\frac{1}{\sqrt{2 \pi}} \sum_{0<|j| \leq \mathrm{C}} w_{j} e^{\mathrm{i} j x}, \quad w_{H}:=\Pi_{H} w:=\frac{1}{\sqrt{2 \pi}} \sum_{|j|>\mathrm{C}} w_{j} e^{\mathrm{i} j x} .
$$

We write $w=w_{L}+w_{H}$ and we define the norm

$$
\|w\|_{s}^{2}:=H_{\mathbb{C}}^{(2)}\left(w_{L}\right)+\left\|w_{H}\right\|_{\dot{H}^{s}}^{2}
$$

where (see (5.6)

$$
H_{\mathbb{C}}^{(2)}(w)=\int_{\mathbb{T}} \Omega(D) w \cdot \bar{w} d x=\sum_{j \in \mathbb{Z} \backslash\{0\}} \Omega(j) w_{j} \overline{w_{j}} .
$$

Since $\Omega(j)>0, \forall j \neq 0$, and $w_{L}$ is supported on finitely many Fourier modes $0<|j| \leq \mathrm{C}$, we have that, for some constant $C_{s}>0$,

$$
C_{s}^{-1}\|w\|_{s} \leq\|w\|_{\dot{H}^{s}} \leq C_{s}\|w\|_{s},
$$

i.e. the norms $\|\cdot\|_{s}$ and $\|\cdot\|_{\dot{H}^{s}}$ are equivalent. We now prove the estimate (5.27) for the equivalent norm $\|\cdot\|_{s}$.

We first note that, by (5.28), $H_{B N F}^{(3)}(z, \bar{z})=H_{B N F}^{(3)}\left(z_{L}, \bar{z}_{L}\right)$. Therefore $\Pi_{H} \partial_{\bar{z}} H_{B N F}^{(3)}(z, \bar{z})=$ 0 and the equation (1.10) amounts to the system

$$
\begin{cases}\dot{z}_{L}=\mathrm{i} \Omega(D) z_{L}+\mathrm{i} \partial_{\bar{z}} H_{B N F}^{(3)}\left(z_{L}, \bar{z}_{L}\right) & +\Pi_{L}\left(\mathcal{X}_{\geq 3}^{+}(U, Z)\right) \\ \dot{z}_{H}=\mathrm{i} \Omega(D) z_{H} & +\Pi_{H}\left(\mathcal{X}_{\geq 3}^{+}(U, Z)\right)\end{cases}
$$

Moreover since the Hamiltonian $H_{B N F}^{(3)}$ in 1.11) is in Birkhoff normal form, it Poisson commutes with the quadratic Hamiltonian $H_{\mathbb{C}}^{(2)}$ in $(5.29$, i.e.

$$
\left\{H_{B N F}^{(3)}, H_{\mathbb{C}}^{(2)}\right\}=0 \text {. }
$$

We have

$$
\begin{aligned}
& \partial_{t} H_{\mathbb{C}}^{(2)}\left(z_{L}\right) \stackrel{5.311}{=}\left\{H_{B N F}^{(3)}, H_{\mathbb{C}}^{(2)}\right\}+2 \operatorname{Re} \int_{\mathbb{T}} \Omega(D) \Pi_{L}\left(\mathcal{X}_{\geq 3}^{+}(U, Z)\right) \cdot \bar{z}_{L} d x \\
& \stackrel{(5.32)}{=} 2 \operatorname{Re} \int_{\mathbb{T}} \Pi_{L} \Omega(D)\left(\mathcal{X}_{\geq 3}^{+}(U, Z)\right) \cdot \Pi_{L} \bar{z} d x \lesssim_{s}\|z\|_{\dot{H}^{s}}^{4}
\end{aligned}
$$


using that $\left\|\Pi_{L} \Omega(D) \mathcal{X}_{\geq 3}^{+}\right\|_{\dot{H}^{0}} \lesssim_{s}\|z\|_{\dot{H}^{s}}^{3}$ by item (2) of Theorem 1.1, Moreover, since $\Pi_{H}$ and $\Pi_{L}$ project on $L^{2}$-orthogonal subspaces,

$$
\begin{aligned}
\partial_{t}\left\|z_{H}\right\|_{\dot{H}^{s}}^{2} & =\partial_{t}\left(|D|^{s} z_{H},|D|^{s} z_{H}\right)_{L^{2}} \stackrel{\sqrt[5.31]{=}}{=} 2 \operatorname{Re} \int_{\mathbb{T}}|D|^{s} \Pi_{H}\left(\mathcal{X}_{\geq 3}^{+}(U, Z)\right) \cdot|D|^{s} \Pi_{H} \bar{z} d x \\
& =2 \operatorname{Re} \int_{\mathbb{T}}|D|^{s} \mathcal{X}_{\geq 3}^{+}(U, Z) \cdot|D|^{s} \bar{z} d x-2 \operatorname{Re} \int_{\mathbb{T}}|D|^{s} \Pi_{L}\left(\mathcal{X}_{\geq 3}^{+}(U, Z)\right) \cdot|D|^{s} \Pi_{L} \bar{z} d x \\
& \stackrel{\varliminf_{s}}{\lesssim_{s}}\|z\|_{\dot{H}^{s}}^{4}+\left\|\Pi_{L} \mathcal{X}_{\geq 3}^{+}\right\|_{\dot{H}^{s}}\left\|\Pi_{L} z\right\|_{\dot{H}^{s}} \lesssim_{s}\|z\|_{\dot{H}^{s}}^{4}
\end{aligned}
$$

by item (2) of Theorem 1.1. Integrating in $t$ the inequalities (5.33), (5.34), we deduce

$$
\|z(t)\|_{s}^{2} \lesssim_{s}\|z(0)\|_{s}^{2}+\int_{0}^{t}\|z(\tau)\|_{\dot{H}^{s}}^{4} d \tau
$$

which, together with the equivalence (5.30), implies (5.27).

Conclusion of the Proof of Theorem 1.2. Consider initial data $\left(\eta_{0}, \psi_{0}\right)$ satisfying (1.15) with $s \gg 1$ given by Theorem 1.1. Classical local existence results imply that

$$
(\eta, \psi) \in C^{0}\left(\left[0, T_{\mathrm{loc}}\right], H_{0}^{s+\frac{1}{4}}(\mathbb{T}, \mathbb{R}) \times \dot{H}^{s-\frac{1}{4}}(\mathbb{T}, \mathbb{R})\right)
$$

for some $T_{\text {loc }}>0$ and thus (1.8) holds with $\bar{\varepsilon}=2 \varepsilon$ and $T=T_{\text {loc }}$. A standard bootstrap argument based on the energy estimate (5.27) (see for instance Proposition 7.6 in [7]) implies that the solution $z(t)$ of 1.10 can be extended up to a time $T_{\varepsilon}:=c_{0} \varepsilon^{-2}$ for some $c_{0}>0$, and satisfies

$$
\sup _{t \in\left[0, T_{\varepsilon}\right]}\|z(t)\|_{\dot{H}^{s}} \lesssim_{s} \varepsilon
$$

We deduce (1.16) by (5.35), the equivalence (5.26), and going back to the original variables $(\eta, \psi)$ by (3.3) and (3.1).

\section{References}

[1] Alazard T., Burq N., Zuily C., On the water-wave equations with surface tension. Duke Math. J., 158, 413-499, 2011.

[2] Alazard T., Delort J-M., Global solutions and asymptotic behavior for two dimensional gravity water waves. Ann. Sci. Éc. Norm. Supér., 48, no. 5, 1149-1238, 2015.

[3] Alazard T., Métivier G., Paralinearization of the Dirichlet to Neumann operator, and regularity of the three dimensional water waves. Comm. Partial Differential Equations 34, no. 10-12, 1632-1704, 2009.

[4] Ambrose D.M., Well-posedness of vortex sheets with surface tension. SIAM J. Math. Anal. 35, no. 1, 211-244, 2003. 
[5] Baldi P., Berti M., Haus E., Montalto R., Time quasi-periodic gravity water waves in finite depth, Inventiones Math. 214 (2), 739-911, 2018.

[6] Berti M., Delort J.-M., Almost global solutions of capillary-gravity water waves equations on the circle. UMI Lecture Notes 2018, ISBN 978-3-319-99486-4.

[7] Berti M., Feola R., Pusateri F., Birkhoff normal form and long time existence for periodic gravity water waves. arXiv:1810.11549, 2018.

[8] Berti M., Montalto R., Quasi-periodic standing wave solutions of gravity-capillary water waves, MEMO 891, to appear on Memoirs AMS.

[9] Beyer K., Günther M., On the Cauchy problem for a capillary drop. I. Irrotational motion. Math. Methods Appl. Sci. 21, no. 12, 1149-1183, 1998.

[10] Christianson H., Hur V., Staffilani G., Strichartz estimates for the water-wave problem with surface tension. Comm. Partial Differential Equations 35, no. 12, 2195-2252, 2010.

[11] Coutand D., Shkoller S., Well-posedness of the free-surface incompressible Euler equations with or without surface tension. J. Amer. Math. Soc. 20, no. 3, 829-930, 2007.

[12] Craig W., An existence theory for water waves and the Boussinesq and Korteweg-de Vries scaling limits. Comm. Partial Differential Equations, 10, no. 8, 787-1003, 1985.

[13] Craig W., Sulem C., Numerical simulation of gravity waves. J. Comput. Phys., 108(1):73-83, 1993.

[14] Craig W., Sulem C., Normal form transformations for capillary-gravity water waves. Fields Inst. Commun., 75, pages 73-110, 2015.

[15] Deng Y., Ionescu A. D., Pausader B., Pusateri F., Global solutions for the 3D gravitycapillary water waves system. Acta Math. 219, no. 2, 213-402, 2017.

[16] Feola R., Iandoli F., Long time existence for fully nonlinear NLS with small Cauchy data on the circle, arXiv:1806.03437, 2018.

[17] Germain P., Masmoudi N., Shatah J., Global solutions for the gravity water waves equation in dimension 3. Ann. of Math. (2), 175, 691-754, 2012.

[18] Germain P., Masmoudi N., Shatah J., Global solutions for capillary waves equation in dimension 3. Comm. Pure Appl. Math., 68, no. 4, 625-687, 2015.

[19] Harrop-Griffiths B., Ifrim M., Tataru D., Finite depth gravity water waves in holomorphic coordinates. Ann. PDE 3, no. 1, Art. 4, 102 pp., 2017.

[20] Hunter J., Ifrim M., Tataru D., Two dimensional water waves in holomorphic coordinates. Comm. Math. Phys. 346, 483-552, 2016. 
[21] Ifrim M., Tataru D., Two dimensional water waves in holomorphic coordinates II: global solutions. Bull. Soc. Math. France 144, 369-394, 2016.

[22] Ifrim M., Tataru D., The lifespan of small data solutions in two dimensional capillary water waves. Arch. Ration. Mech. Anal. 225, no. 3, 1279-1346, 2017.

[23] Ionescu A., Pusateri F., Global solutions for the gravity water waves system in $2 d$. Inventiones Math., 199, 3, 653-804, 2015.

[24] Ionescu A., Pusateri F., Global regularity for $2 d$ water waves with surface tension. Mem. Amer. Math. Soc., 256, no. 1227, v+124, 2018.

[25] Ionescu A., Pusateri F., Long-time existence for multi-dimensional periodic water waves, arXiv:1807.02932, 2018.

[26] Lannes D., The water waves problem: mathematical analysis and asymptotics. Mathematical Surveys and Monographs, 188, 2013.

[27] Nalimov V. I., The Cauchy-Poisson problem. Dinamika Splosn. Sredy Vyp. 18 Dinamika Zidkost. so Svobod. Granicami, 10-210, 254, 1974.

[28] Shatah J., Zeng C., Local well-posedness for the fluid interface problem. Arch. Ration. Mech. Anal. 199, no. 2, 653-705, 2011.

[29] Totz N., Wu S., A rigorous justification of the modulation approximation to the $2 D$ full water wave problem. Comm. Math. Phys. 310, no. 3, 817-883, 2012.

[30] Wu S., Well-posedness in Sobolev spaces of the full water waves problem in 2-D. Inventiones Math. 130(1), 39-72, 1997.

[31] Wu S., Well-posedness in Sobolev spaces of the full water waves problem in 3-D. J. Am. Math. Soc. 12(2), 445-495, 1999.

[32] Wu S., Almost global wellposedness of the 2-D full water wave problem. Inventiones Math., 1, 177, 45-135, 2009.

[33] Wu S., Global well-posedness of the 3-D full water wave problem. Inventiones Math., 1, 184, 125-220, 2011.

[34] Yosihara H., Gravity waves on the free surface of an incompressible perfect fluid of finite depth. Publ. Res. Inst. Math. Sci. 18, 49-96, 1982.

[35] Zakharov V.E., Stability of periodic waves of finite amplitude on the surface of a deep fluid. Zhurnal Prikladnoi Mekhaniki i Teckhnicheskoi Fiziki 9, no.2, 86-94, 1969.

MASSIMILIANO BERTI, SISSA, Trieste, berti@sissa.it ROBERTO FEOLA, UNIVERSITY OF NANTES, roberto.feola@univ-nantes.fr LUCA FRANZOI, SISSA, Trieste, luca.franzoi@ sissa.it 